\title{
Refinements in biostratigraphy of the foraminiferal zone MFZ11 (late early Viséan, Mississippian) in the Cebeciköy Limestone (İ stanbul Terrane, NW Turkey) and palaeogeographic implications
}

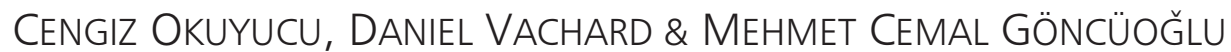

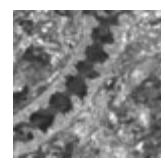

\begin{abstract}
The Cebeciköy Limestone from the Variscan flysch of the İstanbul Terrane is assigned to the foraminiferal MFZ11 zone (late early Viséan, formerly V2a). This zone is essentially the range zone of Uralodiscus rotundus. Additional bioevents are proposed for characterizing the upper part of this biozone (MFZ11B subzone): the LAD of Eoendothyranopsis and the FAD of Pararchaediscus and Conilidiscus. The majority of foraminifers and algae, belonging locally to MFZ11 are well-known, except for the following taxa: Issinella luteotubulifomis sp. nov., I. enormis sp. nov., Vicinesphaera parasqualida sp. nov., Planogloboendothyra modesta sp. nov. and Endothyra irinaeformis sp. nov. The Istanbul Terrane is connected with the southern branch of the Palaeotethys, whereas the Zonguldak Terrane is connected with the northern branch of this one. $\bullet$ Key words: Viséan, Turkey, İstanbul Terrane, foraminiferal zone MFZ11, biostratigraphy, foraminifers, algae incertae sedis, systematics, palaeogeography.
\end{abstract}

OKUYUCU, C., VACHARD, D. \& GÖNCÜOĞLU, M.C. 2013. Refinements in biostratigraphy of the foraminiferal zone MFZ11 (late early Viséan, Mississippian) in the Cebeciköy Limestone (İstanbul Terrane, NW Turkey) and palaeogeographic implications. Bulletin of Geosciences 88(3), 621-645 (14 figures). Czech Geological Survey, Prague. ISSN 1214-1119. Manuscript received September 10, 2012; accepted in revised form January 15, 2013; published online April 26, 2013; issued July 3, 2013.

Cengiz Okuyucu (corresponding author), Selcuk University, Faculty of Engineering, Department of Geological Engineering, Selcuklu, Konya, Turkey; okuyucucengiz@gmail.com•Daniel Vachard, Université Lille 1, UMR CNRS 8217: Géosystèmes, Bâtiment SN5, 59655 Villeneuve d'Ascq cedex, France; Daniel.Vachard@univ-lille1.fr・Mehmet Cemal Göncüoğlu, Middle East Technical University (METU), Department of Geological Engineering, 06531 Ankara, Turkey; mcgoncuoglu@gmail.com

The closure of the Rheic Ocean during the Middle-Late Palaeozoic in Europe resulted in the formation of the Variscan Belt. In western and central Europe, the rock units of this belt and their stratigraphic features have been studied in detail. Towards eastern Europe, the Variscan Belt can be followed in the Balkan (Yanev 1993, 1997, 2000), NW Anatolian (Göncüoğlu et al. 1997, 2006) and Caucasian (Yanev \& Adamia 2010) terranes (Fig. 1). In NW Anatolia, Variscan successions are known since the mid $19^{\text {th }}$ century as the "Palaeozoic of İstanbul" (Verneuil 1836-1837, Strickland 1840, Tschihatcheff 1854). The Mississippian limestones are widespread in Turkey (e.g., Çatal et al. 1978, Lys 1988, Leven \& Okay 1996, Görür et al. 1997, Kalvoda 2003). The Viséan foraminifers and algae are relatively well-known in southern Turkey (Güvenç 1965, Altıner 1981, Altıner \& Özgül 2001, Okuyucu \& Vachard 2006, Göncüoğlu et al. 2007) but their knowledge remains poor in central and northern Turkey (Kaya \& Mamet 1971; Dil 1975, 1977; Dil et al. 1977; Caridroit et al. 1997; Pille et al. 2010).

In the İstanbul Terrane, the Cebeciköy Limestone yielded the oldest precise dating (Kaya \& Mamet 1971), with the characterization of "V2a" foraminifers. The V2a, originally defined as a middle Viséan division, is currently connected with the late early Viséan. Our goals in this paper have been: (1) to provide a detailed analysis of the foraminifers and algae of Cebeciköy; (2) to verify that the Cebeciköy Limestone contained the MFZ11 biozone (Poty et al. 2006, Hance et al. 2011) corresponding roughly to the ancient "V2a"; (3) to revise the eponymous locality of Cebeciköy in order to provide a new detailed analysis of this biozone; (4) to discuss if the subzonation into MFZ11A and MFZ11B existed also locally; (5) to provide some elements of a palaeogeographical analysis of the northern terranes of Turkey. 


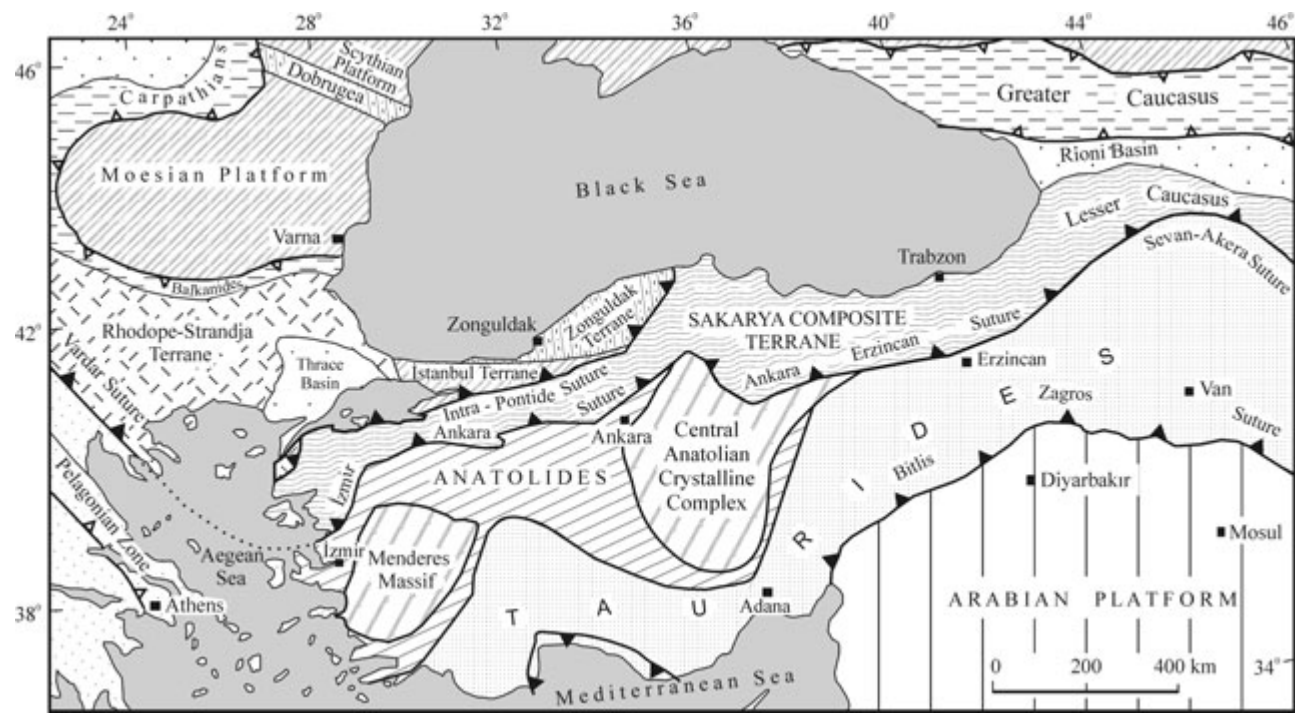

Figure 1. Geological map of Turkey and surrounding area (modified after Göncüoğlu et al. 1997 and Okay \& Tüysüz 1999).

\section{Geological setting of Cebeciköy Limestone}

The oldest Palaeozoic successions of the İstanbul Terrane are Early? Ordovician fluviatile (Kocatöngel and Kurtköy formations) to lagoonal sediments (Kaya, unpublished TPAO report 1982, Özgül 2012). The earliest marine transgression (Aydos Formation) occurred during the endOrdovician. Its platform margin environments prevailed up to the late Middle Devonian (Fig. 2: Gözdağ, Dolayoba, Istinye and Kartal formations). The macrofaunal and microfaunal data of this period indicate that the palaeogeographic position of the İstanbul Terrane remained unchanged compared to the Central European Variscan basins (Haas 1968, Kalvoda et al. 2003, Dojen et al. 2004, Yanev et al. 2006). From Late Devonian onwards, a rapid subsidence and deposition of slope-type sediments (Fig. 2, Büyükada Fm.) started (Önalan 1981). The latest Devonian-earliest Carboniferous interval is characterized by deposition of radiolarian cherts (Göncüoğlu et al. 2004, Noble et al. 2008) in basinal conditions (Fig. 2, Ayineburnu Formation). They are followed by a rapid shallowing-upward sequence, composed of plant-bearing proximal turbidites (Kaya 1969, 1971). They grade into a more than 2000 m-thick succession of flyschoid sediments of the Trakya Formation, originally named as the "Thrazische Serie" by Penck (1919). Except for a few Lower Carboniferous foraminifers found in shally lenses (Kaya \& Mamet 1971) in the lower part of the formation, these mass-flow deposits are barren. The only age data had been obtained from the randomly distributed carbonate lenses named as the Cebeciköy Limestone Member (Yalçınlar 1951, 1954).

In this study, the stratigraphy, depositional features as well as the foraminiferal content of the Cebeciköy Limestone in its type locality to the west of Bosphorus (Fig. 3) will be reviewed and correlated with other limestone occurrences east of the Bosphorus. It should be noted, how- ever, that the original succession described by Yalçınlar (1954) has been completely quarried and his fossil collection is no longer available. Thus, the recently sampled section very probably differs in lithological detail and also in the exact age of the upper and lower boundaries from the earlier one. The distribution of foraminiferal fauna and systematic positions are revised and compared with the published data of the Cebeciköy Limestone. Finally, the fossil content of these intra-flysch deposits is compared with that of the coeval formations in central and western Europe and Alborz (Iran) to search for a palaeogeographic/palaeobiogeographic relationship.

Lithostratigraphy. - The Trakya Formation, in general, comprises a very thick package of turbiditic sandstones and siltstones with minor conglomerate and shale interlayers, and a limestone member (Fig. 2, Cebeciköy Limestone). The formation, with lateral facies changes, covers vast areas on both sides of the Bosphorus. Özgül et al. (2005) subdivided it into four members; from bottom to top: 1) Acıbadem Mb., 2) Cebeciköy Limestone Mb., 3) Kartaltepe Mb., and 4) Küçükköy Mb. (Fig. 2).

The Acrbadem $\mathrm{Mb}$. consists of silty shales with thin siltstone and fine-grained sandstone interlayers and is transitional to the underlying radiolarian cherts with phosphate nodules (Baltalimanı Fm). The nodules, rich in radiolarians, were recently dated as "middle to late" Tournaisian by Noble et al. (2008), although these stratigraphic subdivisions are now obsolete in the stratotypic Belgian area (Poty, pers. com.). Regionally, it is better developed on the east side of the Bosphorus, with a type locality situated in Acıbadem (Kaya 1971). In the Gebze-Denizliköy area, it shows a $2-15 \mathrm{~cm}$ thick ostracod-rich black limestone with shale interlayers (Göncüoğlu et al., unpublished MTA report, 2006). Upwards, it is represented by greenish-grey muscovite-rich quartz-arenites with laminar siltstone. In 
Figure 2. A - Silurian-Carboniferous series of the İstanbul Terrane (after Gedik et al., unpublished MTA report, 2005, Özgül et al. 2005, Göncüoğlu et al. 2006). • B - measured section of Cebeciköy Limestone Member with sample locations. Legend: $\mathrm{a}-$ shale and limestone alternation; $\mathrm{b}$ - limestone, dolomitic limestone and shale alternation; c - reefal limestone; $\mathrm{d}$ - limestone; $\mathrm{e}$ - thick nodular clayey limestone and shale; f - shale, sandstone and limestone alternation; $\mathrm{g}$ - cherty limestone; $\mathrm{h}$ - siliceous shale and chert alternation; i - thin nodular limestone and shale alternation; $\mathrm{j}$ - radiolarite with nodular phosphate, chert and shale; $\mathrm{k}$ - turbiditic sandstone and shale; 1 - granites.

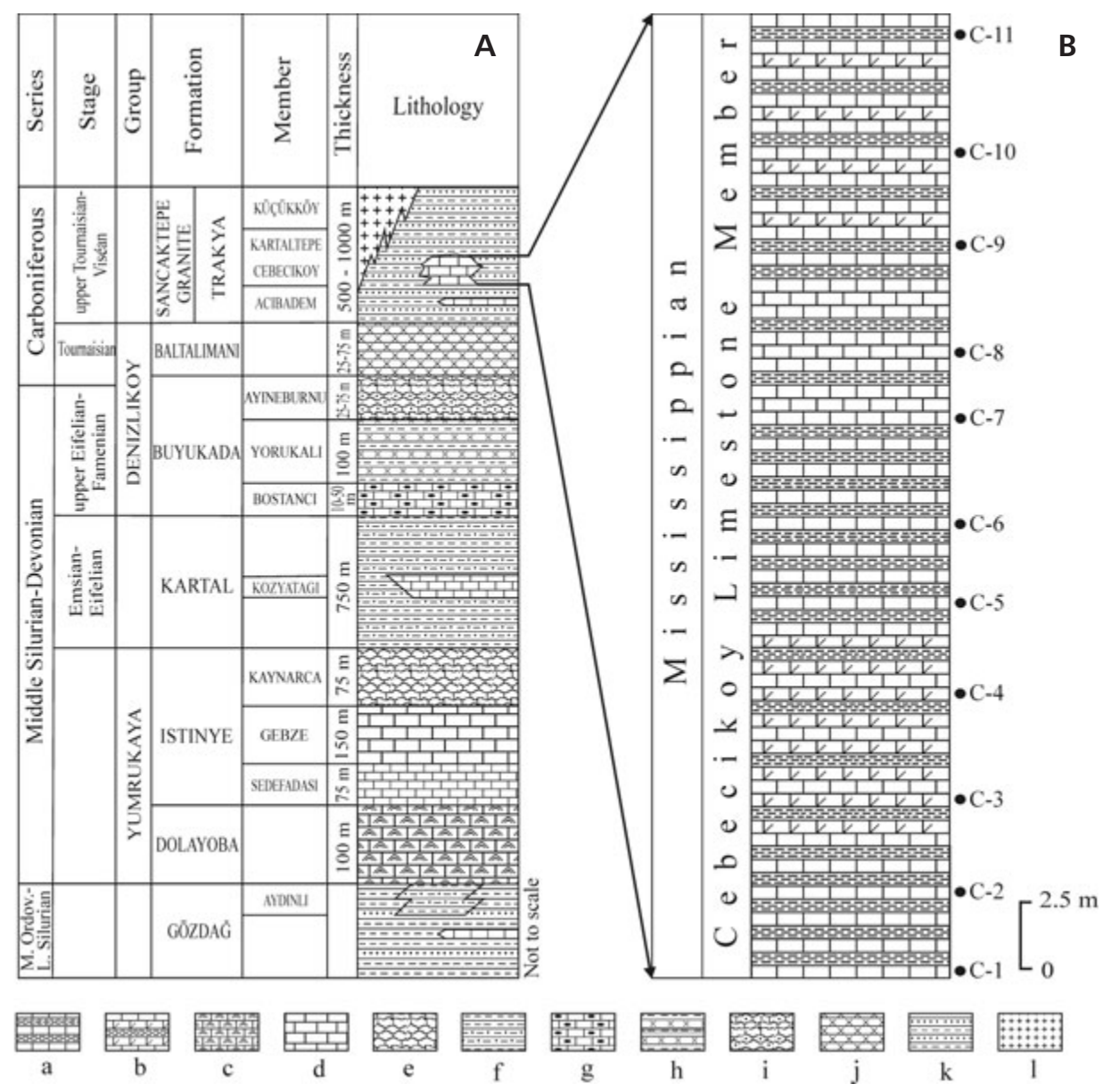

of the Triassic Kapaklı Fm. The Küçükköy Mb. includes medium- to coarse-grained channel-fill-type conglomerates in different stratigraphic positions. The pebbles are dominated by quartz-arenite, quartzite, arkose, lydite, metamorphic and rhyolitic rock fragments together with mineral clasts such as igneous and metamorphic quartz and feldspars. Flute-casts, load-casts and laminations are common features. The member is affected by deformation and includes open to tight folding.

Microfacies. - The microfacies are composed of bioclastic packstone $(\mathrm{C} 1, \mathrm{C} 8-\mathrm{C} 11)$, bioclastic wackestone (C5-C7) and poorly fossiliferous dolomicrosparite (C2-C4). Among the bioclasts, cyanobacteria are very rare, true green and red algae absent, moravamminids always frequent, aoujgaliids rare or very rare, and foraminifers always present, more or less diversified and abundant (Fig. 4).

Therefore, these microfacies display a mixed character between heterozoan and photozoan assemblages. Three explanations can be proposed: (1) There are influxes of cool deep water currents (James 1997, Samankassou 2002) on a subtropical to warm temperate (30-40 of latitude) shallow carbonate platform (Gallagher 1998, Pille 2008). (2) These assemblages might be typical of the pre-LPIA (Late 


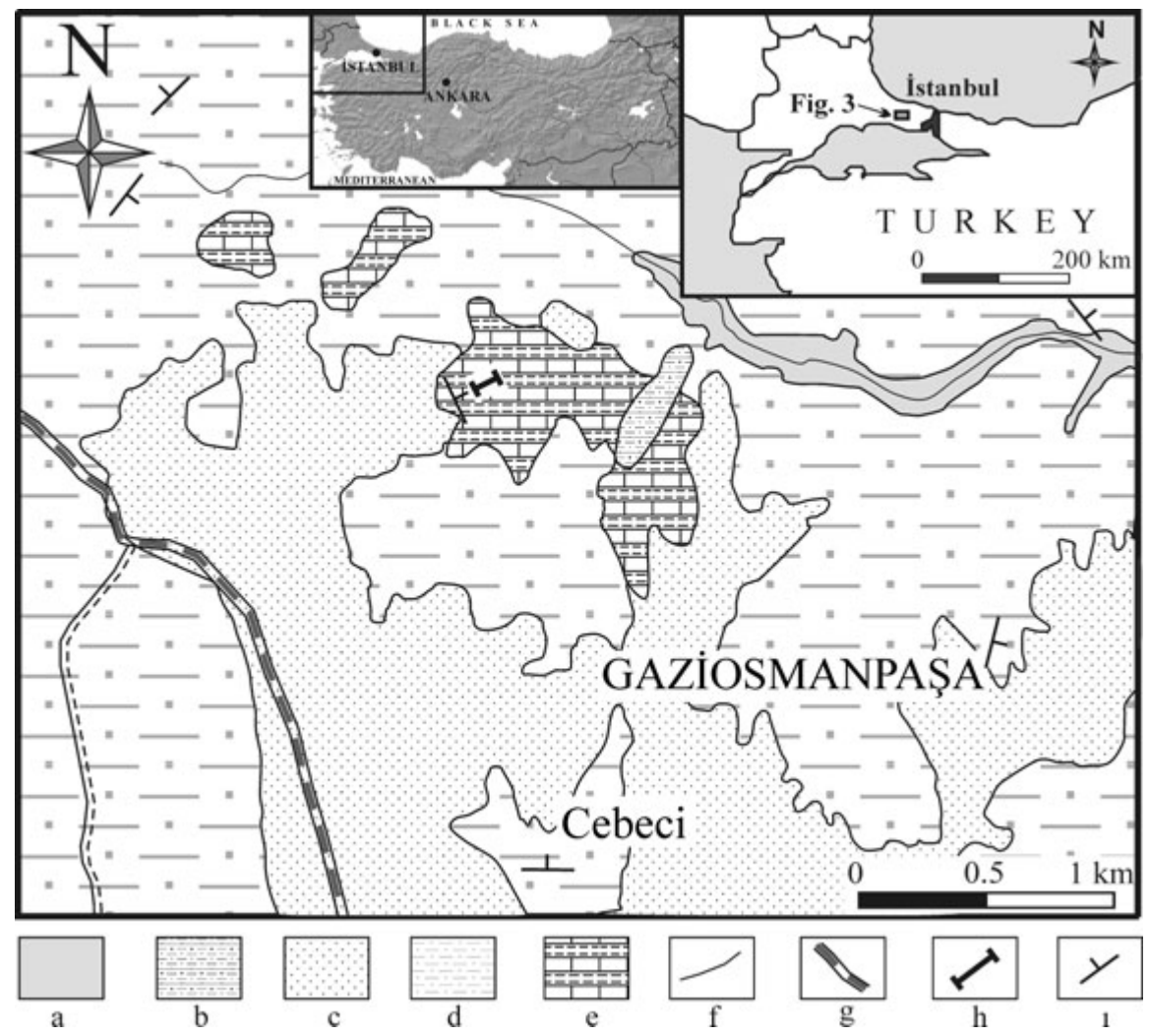

Figure 3. Geological map of the study area (simplified after Gedik et al., unpublished MTA report, 2011). Legend: a - Alluvium, b - Belgrad Formation (Plio-Quaternary); c - Güngören Formation (Upper Miocene), d - Trakya Formation (upper Tournaisian-Viséan), e - Cebeciköy Limestone Member (late early Viséan), f - stream, g - main road, $\mathrm{h}$ - line of logged section, $\mathrm{i}-$ bedding.

Palaeozoic Ice Age) period, where the climate was poorly characterized (Kalvoda 2002) and/or the photozoan carbonate factory with foraminifers occupied a narrow belt (Kalvoda et al. 2011). (3) The assemblages are greatly influenced by the first regional phase of cooling of this LPIA, coevally identified in northern Iran (Termier et al. 1977).

\section{The biozone MFZ11 and its subdivisions}

The biozone MFZ11 of Poty et al. (2006) is situated between the base of the range-zone of Uralodiscus rotundus and the base of the interval-zone of Pojarkovella nibelis (Durkina). The foraminiferal guide-fossil is Uralodiscus rotundus or occasionally the assemblage of Uralodiscus rotundus and Viseidiscus primaevus (e.g., Kagarmanov \& Donakova 1990, Einor 1996). In the Belgian stratotypes, MFZ11 is represented by the Neffe Limestone (Conil \& Naum 1976, Conil et al. 1977, Hance 1988).

The definition of MFZ11 is more precise than that of previous subdivisions, more or less synonymous. The

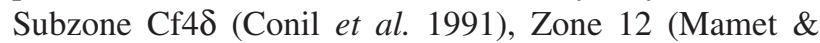
Skipp 1970, Mamet 1974), Zone 10 (Kalvoda 1983), Zone 3.3.13 (Kalvoda 2002), Arundian (which covers in fact MFZ10 and MFZ11; Poty, personal communication), late Moliniacian, Bobrikovsky, Ilychsky, Ust-Grekhovsky, Karakiinsky, and Shishikhinsky regional stages and substages in England, Belgium and Russia, as well as the base of the Jiusi Formation in South China, are more or less equivalent (Malakhova 1975; Mikhno \& Balakin 1975; Kagarmanov \& Donakova 1990; Marfenkova 1991; Einor 1996; Kalvoda 1999; Kulagina et al. 2003; Hecker 2002, 2009; Hance et al. 2011). The subzones $\mathrm{C}_{1}{ }^{\mathrm{v}} \mathrm{d}_{1}-\mathrm{C}_{1}{ }^{\mathrm{v}} \mathrm{d}_{2}$ of Ukraine are also more or less contemporaneous (Vdovenko 1980, 2001; Hecker 2009; Davydov et al. 2010).

The radiometric age of MFZ11 can be currently established between ca 345 and 344 My (Menning et al. 2006, Davydov et al. 2010).

The biozone MFZ 11 is also characterized by numerous FAD (first appearance datum) and LAD (last appearance datum) of foraminifers: (1) LAD of Eoendothyranopsis; (2) FAD of Paraarchaediscus; (3) FAD of various subspecies of Uralodiscus rotundus; (4) FAD of Cribranopsis; (5) FAD of Conilidiscus; (6) LAD of Eoparastaffella; (7) LAD of Pseudolituotubella; (8) LAD of Condrustella; (9) LAD of Eotextularia (Hance et al. 1994, 2011; Poty et al. 2006). The MFZ11 algae in Belgium are marked by: (1) a general rarity of the codiaceans, dasycladaceans and solenoporaceans (Hance 1988, Devuyst 2006); (2) the FO of bilayered Koninckopora (Conil \& Groessens 1974, Hance 1988, Hance et al. 1994); (3) the abundance of Luteotubulus and absence of true Issinella (Hance 1988, Vachard 1994); (4) a lineage of Palaeoberesella to Exvotarisella is poorly documented.

Recent data in South China (Hance et al. 2011) permitted to subdivide MFZ11 in two subzones MFZ11A and 
MFZ11B; the second of which is characterized by the FAD of rare Pojarkovella sp. The FO (first occurrence) of this genus in Belgium, with the species $P$. nibelis (Durkina), is an index of the MFZ12 zone. Some other references confirm this FO of Pojarkovella, before its acme-zone, in Tarim and Alborz (Brenckle 2004: sample 3, Brenckle et al. 2009: sample 807).

The biozone MFZ11 in Cebeciköy. - In Cebeciköy, we found the following markers of the stratotypic Belgian area: Uralodiscus rotundus, Eotextularia diversa, Paraarchaediscus, Conilidiscus, Pseudolituotubella, Eoendothyranopsis, Eoparastaffella, Spinoendothyra, and Endospiroplectammina (Fig. 4). Conversely, some important Belgian taxa (Hance 1988, Conil et al. 1989, Hance et al. 1994, Devuyst et al. 2006) are absent in Cebeciköy: Eogloboendothyra or true Globoendothyra, Pseudolituotuba, Spinobrunsiina (= ?Pseudoplanoendothyra), Paraendothyra, Granuliferella, Latiendothyranopsis menneri solida (Conil \& Lys), Endolaxina laxa (Conil \& Lys) emend. Hance, Hou \& Vachard, Urbanella (Brenckleites) fragilis (Lipina) emend. Hance, Hou \& Vachard, Florennella, Dainella, Bessiella and Valvulinella. Except for Dainella and Pseudoplanoendothyra, the Cebeciköy assemblages have many common taxa with the Orenburg area of Russia (Kulagina et al. 1988).

The foraminiferal assemblages summarized herein (Fig. 4) differ from those identified by Kaya \& Mamet (1971) in the Cebeciköy Limestone. In fact, these latter authors dated equivalents of zones MFZ12 and MFZ13. Probably, most of parts of these possible MFZ12 and MFZ13 levels of Cebeciköy Limestone were completely destroyed because of quarry works. However, our assemblages are relatively similar to that indicated by Mamet (1973) for a Heybeliada Limestone assigned by this author to the middle early Viséan (i.e., MFZ10 sensu Poty et al. 2006). Consequently, some mistakes in the field-sampling and or thin/sections numbering occurred probably during the ancient studies.

As the archaediscids are absent in the eponymous localities of the MFZ11B subzone in South China, we propose, thanks to our Cebeciköy data, to add the FAD of Paraarchaediscus and Conilidiscus (Fig. 5) as a characteristic of the base of MFZ11B in western and central Palaeotethys. Three data confirm this interpretation: (1) The joint occurrence of Pojarkovella and Uralodiscus was reported in Moravia by Kalvoda (1990), (2) in Tarim (NW China), Brenckle (2004; text-fig. 3) indicated coeval FO of Pararchaediscus and Pojarkovella (in his samples 2 and 3), (3) in Alborz (N Iran), a FO of Pojarkovella is located between the FO and LO of Uralodiscus (Brenckle et al. 2009, text-figs 7, 8). Finally, the FAD of Pojarkovella sp. can currently characterize the base of MFZ11B in eastern Europe and Middle and Far-East; those of Paraarchaediscus spp. and Conilidiscus spp. characterize this one in Europe and Near-East, where the migration of Pojarkovella is delayed.
Correlatively with these FAD of Paraarchaediscus and Conilidiscus, the assemblages of Cebeciköy indicate that the LAD of Eoendothyranopsis occurs probably near or at the MFZ11A-MFZ11B boundary (Fig. 5).

Among the algae, the bilayered Koninckopora are not present in Cebeciköy. True Issinella dwelt in Turkey, whereas they are replaced by Luteotubulus in Belgium in MFZ11 (Vachard 1994, Hance 1988). The lineage of Palaeoberesella to Exvotarisella might provide another biostratigraphic criterion in Turkey, due to its relative richness; nevertheless, the distribution of the Palaeoberesella lahuseni-Exvotarisella index assemblage in Russia spreads over a long interval of time, including the MFZ11 and MFZ12 equivalents (Ivanova \& Bogush 1992).

In the Central Tauride Belt (southern Turkey), the biozones VT2a and VT2b (Altıner \& Özgül 2001) might correspond to MFZ11A-B; indeed, they contain particularly Uralodiscus rotundus, Latiendothyranopsis menneri solida, (Para?)-archaediscus, Eoparastaffella simplex, Pseudolituotubella sp., and Eotextularia diversa. Nevertheless, they are followed by the biozone VT3, which still contains Latiendothyranopsis menneri solida, Uralodiscus rotundus, Paraarchaediscus and Eoparastaffella simplex. If some Uralodiscus persist in MFZ12 in South China with many species of Archaediscus at concavus stage and rare species at involutus stage (Hance et al. 2011), the LAD of Eoparastaffella simplex is everywhere located at the top of MFZ11. Consequently, we suggest that VT2a is coeval with MFZ11A, and VT2b+VT3 coeval with MFZ11B.

\section{Systematic palaeontology}

Abbreviations. - L - length, $\mathrm{H}$ - height, D - outer diameter, $\mathrm{d}$ - inner diameter, $\mathrm{s}$ - wall thickness, $\mathrm{w}$ - width, $\mathrm{w} / \mathrm{D}$ - ratio width to outer diameter, $\mathrm{p}$ - proloculus diameter, $\mathrm{n}$ - number of whorls, $\mathrm{h}$ - height of last whorl or last chamber.

\section{Algae incertae sedis}

Class Algospongia Termier, Termier \& Vachard, 1977 emend. Vachard \& Cózar, 2010

Order Moravamminida Pokorny, nom. translat. Termier, Termier \& Vachard, 1975, emend. Vachard \& Cózar, 2010 Suborder Moravamminina Pokorny, 1951, nom. translat.

Vachard \& Cózar, 2010

Family Issinellidae Deloffre, 1987, emend. Vachard \& Cózar, 2010

Genus Issinella Reitlinger, 1954, emend. Vachard \& Cózar, 2010

Type species. - Issinella devonica Reitlinger, 1954. 


\begin{tabular}{|c|c|c|c|c|c|c|c|c|c|c|c|}
\hline \multirow[t]{2}{*}{ TAXA } & \multicolumn{11}{|c|}{ SAMPLES } \\
\hline & $\mathrm{C} 1$ & $\mathrm{C} 2$ & $\mathrm{C} 3$ & $\mathrm{C} 4$ & $\mathrm{C} 5$ & C6 & $\mathrm{C} 7$ & $\mathrm{C} 8$ & C9 & $\mathrm{C} 10$ & $\mathrm{C} 11$ \\
\hline Girvanella wetheredii Chapman, 1908 & & & & & $\varepsilon$ & & & $\varepsilon$ & & & \\
\hline Issinella ex gr. devonica Reitlinger, 1954 & + & + & + & + & $\varepsilon$ & + & + & $\varepsilon$ & & $\varepsilon$ & \\
\hline Issinella luteotubuliformis sp. nov. & & & & & & & & & ++ & + & \\
\hline Issinella enormis sp. nov. & & & & & & & & & & & ++ \\
\hline Evlania scabrosa Vachard, 1980 & & & & & & & & & $\varepsilon$ & $\varepsilon$ & \\
\hline Pseudokamaena cf. armstrongi Mamet in Petryk \& Mamet, 1972 & $\varepsilon$ & & & & & & & $\varepsilon$ & & & \\
\hline Kamaena pirleti Mamet \& Roux, 1974 & + & + & + & + & & & & $\varepsilon$ & + & & \\
\hline Palaeoberesella lahuseni (Möller, 1879) Mamet \& Roux, 1974 & + & + & + & + & $\varepsilon$ & $\varepsilon$ & $\varepsilon$ & $\varepsilon$ & + & $\varepsilon$ & \\
\hline Palaeoberesella sp. 1 & $\varepsilon$ & & & & $\varepsilon$ & & $\varepsilon$ & & & & \\
\hline Exvotarisella index (Ehrenberg, 1854, emend. Möller, 1879) Mamet \& Roux, 1974 & + & + & + & + & $\varepsilon$ & & $\varepsilon$ & $\varepsilon$ & & $\varepsilon$ & $\varepsilon$ \\
\hline Stacheoides cf. tenuis Petryk \& Mamet, 1972 & $\varepsilon$ & & & & & $\varepsilon$ & & $\varepsilon$ & $\varepsilon$ & & \\
\hline Calcisphaera grandis (Lipina, 1950) & & & & & & & & $\varepsilon$ & $\varepsilon$ & $\varepsilon$ & $\varepsilon$ \\
\hline Radiosphaera ponderosa Reitlinger, 1960 & $\varepsilon$ & & & & & $\varepsilon$ & & & $\varepsilon$ & & \\
\hline Eotuberitina reitlingerae Miklukho-Maklay, 1958 & $\varepsilon$ & & & & + & $\varepsilon$ & + & $\varepsilon$ & & & \\
\hline Vicinesphaera parasqualida sp. nov. & ++ & & & & + & $\varepsilon$ & + & $\varepsilon$ & $\varepsilon$ & $\varepsilon$ & + \\
\hline Brunsia pulchra Mikhailov, 1939 & + & & & & & $\varepsilon$ & + & & $\varepsilon$ & & \\
\hline Brunsia spirillinoides (Grozdilova \& Glebovskaya, 1948) & + & & & & & $\varepsilon$ & $\varepsilon$ & & $\varepsilon$ & $\varepsilon$ & + \\
\hline Uralodiscus rotundus rotundus (Chernysheva, 1948a) & + & & & & & $\varepsilon$ & + & $\varepsilon$ & $\varepsilon$ & $\varepsilon$ & \\
\hline Uralodiscus rotundus elongatus (Conil \& Lys, 1964) & + & & & & $\varepsilon$ & $\varepsilon$ & & & $\varepsilon$ & $\varepsilon$ & \\
\hline Uralodiscus rotundus inflatus (Conil \& Lys, 1964) & + & & & & + & + & & $\varepsilon$ & & $\varepsilon$ & $\varepsilon$ \\
\hline Uralodiscus rotundus vastus (Popova, 1970) & & & & & & & & & $\varepsilon$ & $\varepsilon$ & $\varepsilon$ \\
\hline Conilidiscus cf. bucculentus (Conil \& Lys, 1964) & & & & & & & & & $\varepsilon$ & $\varepsilon$ & \\
\hline Paraarchaediscus cf. sumsariensis (Skvortsov, 1965) & & & & & + & & & & & & \\
\hline Paraarchaediscus regularis (Brazhnikova \& Vdovenko, 1973) & & & & & & & & & & $\varepsilon$ & \\
\hline Paraarchaediscus aff. pauxillus (Shlykova, 1951) & & & & & $\varepsilon$ & & & & & & \\
\hline $\begin{array}{l}\text { Earlandia ex gr. elegans (Rauzer-Chernousova \& Reitlinger in Rauzer-Chernousova \& } \\
\text { Fursenko, 1937) }\end{array}$ & + & & & & + & & $\varepsilon$ & & & & \\
\hline Earlandia ex gr. minor (Rauzer-Chernousova, 1948b) & + & & & & + & $\varepsilon$ & + & & & & + \\
\hline $\begin{array}{l}\text { Earlandia ex gr. vulgaris (Rauzer-Chernousova \& Reitlinger in Rauzer-Chernousova \& } \\
\text { Fursenko, 1937) }\end{array}$ & $\varepsilon$ & & & & $\varepsilon$ & $\varepsilon$ & & & & & $\varepsilon$ \\
\hline Magnitella cf. agapovensis (Ivanova, 1988) & & & & & & $\varepsilon$ & $\varepsilon$ & $\varepsilon$ & $\varepsilon$ & $\varepsilon$ & \\
\hline Baituganella ex gr. vulgaris Lipina, 1955 & $\varepsilon$ & & & & & & & & & & \\
\hline Forschia parvula Rauzer-Chernousova, 1948b & $\varepsilon$ & & & & + & $\varepsilon$ & + & & & $\varepsilon$ & \\
\hline
\end{tabular}

Figure 4. Microfauna and microflora of the Cebeciköy Member (to be continued on p. 7). For sample location see Fig. 2.

Diagnosis. - Small or medium sized tubular thallus, rarely bifurcated rarely bearing diaphragms. Wall yellowish, homogeneous (not maculate) with relatively numerous, aspondyl, acrophore perpendicular canalicules.

Remarks. - Some Issinella show rare diaphragms: I. luteotubuliformis sp. nov., I.? ilychensis Ivanova, 1988 and I. devonica (sensu Mamet \& Roux, 1981, pl. 1, figs 6, 7 and sensu Bogush et al., 1990, pl. 12, fig. 9). Luteotubulus Vachard in Vachard et al., 1977, emend. Vachard, 1994 differs by relatively common diaphragms, a maculate wall and more numerous thinner, filiform, curved canalicules in the wall.

Occurrence. - Early Devonian-late Serpukhovian; cosmopolitan.

\section{Issinella ex gr. devonica Reitlinger, 1954}

Figures 6A, 9, 7A-C, 9I, 11, 12T, 13B

Discussion. - Three criteria separate our material from true I. devonica: (a) different dimensions ( $\mathrm{L}=1.000-1.670 \mathrm{~mm}$, $\mathrm{D}=0.125-0.350 \mathrm{~mm}, \mathrm{~d}=0.070-0.150 \mathrm{~mm}, \mathrm{~s}=$ $0.015-0.050$ (rarely 0.070 ) $\mathrm{mm}$; the wall is notably thicker); (b) a specimen seems to show ramified canalicules (Fig. 6C); (c) the type material of I. devonica comes from the late Frasnian and the identifications of this species in the Early Carboniferous are generally erroneous (Mamet \& Roux 1981, Vachard \& Cózar 2010). Issinella grandis Chuvashov, 1965, another species mentioned in the Viséan (Bogush et al. 1990), is larger.

Occurrence. - MFZ11A-B of Cebeciköy. 


\begin{tabular}{|c|c|c|c|c|c|c|c|c|c|c|c|}
\hline \multirow[t]{2}{*}{ TAXA } & \multicolumn{11}{|c|}{ SAMPLES } \\
\hline & $\mathrm{C} 1$ & $\mathrm{C} 2$ & $\mathrm{C} 3$ & $\mathrm{C} 4$ & $\mathrm{C} 5$ & C6 & $\mathrm{C} 7$ & $\mathrm{C} 8$ & C9 & $\mathrm{C} 10$ & $\mathrm{C} 11$ \\
\hline Viseina? sp. & & & & & $\varepsilon$ & & & & & & \\
\hline Planogloboendothyra modesta sp. nov. & + & & & & & & & & & & \\
\hline Pseudolituotubella orientalis (Chernysheva in Dain, 1953) & + & & & & + & $\varepsilon$ & + & & & & \\
\hline Pseudolituotubella aff. tschernyshevi (Dain, 1953) & $\varepsilon$ & & & & + & & & & & & \\
\hline Condrustella cf. modavensis (Conil \& Lys, 1967) & & & & & $\varepsilon$ & $\varepsilon$ & $\varepsilon$ & & & & \\
\hline Eotextularia diversa (Chernysheva, 1948b) & $\varepsilon$ & & & & $\varepsilon$ & $\varepsilon$ & $\varepsilon$ & $\varepsilon$ & & & \\
\hline Eotextularia cf. mongeri Mamet in Mamet et al., 1970 & $\varepsilon$ & & & & & & & & & & \\
\hline Endothyra prisca Rauzer-Chernousova in Rauzer-Chernousova et al., 1936 & $\varepsilon$ & & & & & & & + & & & \\
\hline Endothyra praesimilis (Lin, 1981) & $\varepsilon$ & & & & $\varepsilon$ & & & & & & \\
\hline Endothyra similis amplis Shlykova, 1951 & + & & & & $\varepsilon$ & $\varepsilon$ & $\varepsilon$ & & + & & \\
\hline Endothyra similis inops (Conil \& Lys, 1964) & + & & & & + & + & $\varepsilon$ & $\varepsilon$ & + & & $\varepsilon$ \\
\hline Endothyra paraprisca Shlykova, 1951 & + & & & & $\varepsilon$ & + & + & & + & $\varepsilon$ & \\
\hline Endothyra irinaeformis sp. nov. & $\varepsilon$ & & & & & & & & & & \\
\hline Endothyra acantha (Conil \& Lys, 1964) & & & & & & & $\varepsilon$ & & & & \\
\hline Endothyra gratiodentalis (Lin, 1981) & & & & & & & $\varepsilon$ & & & & \\
\hline Endospiroplectammina cf. conili Lipina, 1970 & $\varepsilon$ & & & & & $\varepsilon$ & $\varepsilon$ & & & & \\
\hline Mediendothyra barzassiensis (Lebedeva, 1954) & + & & & & + & + & + & + & + & $\varepsilon$ & \\
\hline Omphalotis aff. involuta (Brazhnikova, 1956) & $\varepsilon$ & & & & + & $\varepsilon$ & + & + & $\varepsilon$ & & \\
\hline Omphalotis cf. parasamarica (Bogush \& Yuferev, 1962) & & & & & & & & + & + & & $\varepsilon$ \\
\hline Inflatoendothyra aff. multispira (Simonova, 1975a) & $?$ & & & & & & $\varepsilon$ & & & & \\
\hline Latiendothyranopsis? cf. menneri (Bogush \& Yuferev, 1960) & & & & & & $\varepsilon$ & & & & & \\
\hline Plectogyranopsis convexa (Rauzer-Chernousova, 1948a) & $\varepsilon$ & & & & $\varepsilon$ & + & $\varepsilon$ & & & & \\
\hline Plectogyranopsis ampla (Conil \& Lys, 1964) & $\varepsilon$ & & & & & & $\varepsilon$ & & & & \\
\hline Plectogyranopsis cf. dendrei (Conil \& Lys, 1964) & & & & & & & $\varepsilon$ & & & & \\
\hline Eoendothyranopsis? cf. ermakiensis (Lebedeva, 1954) & + & & & & & & & & & & \\
\hline Eoendothyranopsis donica tumefacta Postoyalko, 1975 & + & & & & & & & & & & \\
\hline Tetrataxis immatura Grozdilova \& Lebedeva, 1954 & + & & & & $\varepsilon$ & $\varepsilon$ & $\varepsilon$ & & $\varepsilon$ & $\varepsilon$ & \\
\hline Tetrataxis aff. angusta Vissarionova, 1948 & & & & & $\varepsilon$ & $\varepsilon$ & & & $\varepsilon$ & & $\varepsilon$ \\
\hline Tetrataxis bogushi Ueno \& Nakazawa, 1993 & & & & & $\varepsilon$ & & & & $\varepsilon$ & $\varepsilon$ & \\
\hline Tetrataxis cf. quasiconica Brazhnikova, 1956 & $\varepsilon$ & & & & & & & & & $\varepsilon$ & \\
\hline Mediocris breviscula (Ganelina, 1951) & $\varepsilon$ & & & & & & & $\varepsilon$ & & & \\
\hline Eoparastaffella simplex lata Vdovenko, 1971 & $\varepsilon$ & & & & & $\varepsilon$ & $\varepsilon$ & + & & & \\
\hline Eoparastaffella simplex ovalis Vdovenko, 1954 & + & & & & $\varepsilon$ & $\varepsilon$ & + & $\varepsilon$ & $\varepsilon$ & $\varepsilon$ & $\varepsilon$ \\
\hline
\end{tabular}

\section{Issinella luteotubuliformis sp. nov.}

Figures 6B, C, E, 7D-F

Etymology. - Similar to Luteotubulus by its diaphragms but different by its type of wall and perforations.

Holotype. - Fig. 6.3, sample C-9(5).

Type horizon. - Late early Viséan (MFZ11B).

Type locality. - Cebeciköy (NW Turkey).

Diagnosis. - This unquestionable Issinella (because of the type of perforations) is characterized by its medium size and rare individuals with diaphragms.

Description. - Test regularly cylindrical, medium-sized.
Perforations acrophore relatively wide. Some bifurcated thalli show an angle of $c a 120^{\circ}$ (Fig. 6B). One to four micritic diaphragms per tube; or very rare yellowish diaphragms (Fig. 7E). Inner surfaces always smooth; outer surfaces very rarely faintly serrated (ontogenetically, not diagenetically by stylolitization). No constrictions. Wall homogeneous, yellowish. $\mathrm{L}=$ (rarely 0.750)-1.250-4.750 mm, $\mathrm{D}=(0.215)-0.250-0.500 \mathrm{~mm}, \mathrm{~d}=(0.135)-0.185-0.400 \mathrm{~mm}$, $\mathrm{s}=(0.030)-0.060-0.120 \mathrm{~mm}$.

Material. - Approximately 250 specimens.

Repository of the types. - University Lille 1 collection (USTL1/1097).

Discussion. - This new species differs from I. ex gr. devonica by larger dimensions; from I. enormis sp. nov. by 


\begin{tabular}{|c|c|c|c|c|c|c|c|c|c|}
\hline BIOZONES & SAMPLES & 1 & 2 & 3 & 4 & 5 & 6 & 7 & 8 \\
\hline \multirow{3}{*}{ MFZ11B } & Cl1 & & & & & & & & \\
\hline & C9 & & & & & & & & \\
\hline & C5 & & & & & & & & \\
\hline MFZ11A & $\mathrm{C} 1$ & & & & & & & & \\
\hline
\end{tabular}

Figure 5. Main markers of the subbiozone MFZ11A and MFZ11B in Cebeciköy (Turkey). • 1 - Eoeodothyranopsis ex gr. donica; 2 - Latiendothyranopsis? ex gr. menneri; 3 -Uralodiscus; 4 -Pararchaediscus; 5 - Conilidiscus; 6 - Issinella ex gr. devonica; 7 - Issinella luteotubuliformis sp. nov.; 8 - Issinella enormis sp. nov.

smaller dimensions; from I.? ilychensis by its larger dimensions, the angle of thallus bifurcation, and a more regularly cylindrical growth of the thallus. Luteotubulus licis (Malakhova, 1975) Vachard in Vachard et al., 1977, whose dimensions are similar, has a maculate, slightly constricted and intensively perforated thallus.

Occurrence. - Type locality and type horizon.

Issinella enormis sp. nov.

Figures 6D, 7G-I

Etymology. - Enormis, huge.

Holotype. - Fig. 6D, sample C-11(2).

Type horizon. - Late early Viséan (MFZ11B).

Type locality. - Cebeciköy (NW Turkey).

Diagnosis. - Huge Issinella with few canalicules in the wall and no diaphragms.

Description. - Test regularly cylindrical, but slightly undulating; enormous for the genus. Perforations very rare. No bifurcated thalli have been observed, nor diaphragms. No constrictions. Wall homogeneous, yellowish. $\mathrm{L}=2.500$ to $8.000 \mathrm{~mm}$, $\mathrm{D}=0.775$ to $1.200 \mathrm{~mm}, \mathrm{~d}=$ (rarely 0.260$)-0.385-0.820 \mathrm{~mm}$, $\mathrm{s}=0.150$ to 0.325 (rarely 0.370$) \mathrm{mm}$.
Material. - 120 specimens.

Repository of the types. - University Lille 1 collection (USTL1/1098).

Discussion. - This new species differs from I. ex gr. devonica and I. luteotubuliformis sp. nov. by its very larger dimensions, and from small corallites of syringoporids (Tabulata), by slightly undulating tubes (like the holotype) and some perforations in the wall.

Occurrence. - Type locality and type horizon.

Subphylum Foraminifera d'Orbigny, nom. translat.

Cavalier-Smith

Class Fusulinata Möller, nom. translat. Gaillot \& Vachard Order Parathuramminida Bykova, nom. translat.

Mikhalevich

\section{Genus Vicinesphaera Antropov, 1950}

Vicinesphaera parasqualida sp. nov.

Figures 6F, 9J, K, R, 10H, 11G, 12U, 13D

Etymology. - Similar to V. squalida Antropov, 1950.

Holotype. - Fig. 10H, sample C-1.2[13/2(4)].

Type horizon. - Late early Viséan (MFZ11A).

Type locality. - Cebeciköy Limestone (NW Turkey).

Diagnosis. - A species of Vicinesphaera characterized by a relatively thin wall and few pyramidal protuberances. Occasionally, the wall displays a thin clear central layer.

Description. - The test measures a total length of $0.100-0.350 \mathrm{~mm}$. The internal chamber is remarkably regular, spherical, wide and measures $0.040-0.180 \mathrm{~mm}$. The wall shows two parts: (a) a regular envelope of the internal chamber, the thickness of which is $0.005-0.030 \mathrm{~mm}$; (b) some protuberances, generally one or two per section, pyramidal, with a height of (rarely 0.010)-0.025-0.100 mm. The sections through the regular envelope (a) resemble a lot to Calcisphaera. The wall is microgranular, dark, homogeneous; nevertheless, rare specimens (including the holotype) show a very

Figure 6. Scale bars $=0.5 \mathrm{~mm}$. For the authors of taxa of Figs 6-13, see Fig. 4. $\bullet$ A - Issinella ex gr. devonica. C-1.3(6). $\bullet$ B, C - Issinella luteotubuliformis sp. nov.; B - paratype. C-9.1(9), C - holotype. C-9(5). • D - Issinella enormis sp. nov., holotype. C-11(2). $\bullet$ E - Evlania scabrosa with four sections of Issinella luteotubuliformis sp. nov. (paratypes). C-9(1). • F - Kamaena pirleti with Vicinesphaera parasqualida sp. nov. (paratype; bottom, right?). C-1.3(19). • G - Exvotarisella index with Palaeoberesella lahuseni (top) and scattered Endothyra similis amplis. C-1(22). • H - Pseudokamaena cf. armstrongi. C-1(9). • I, J - Stacheoides cf. tenuis; I - left with Endothyra prisca (centre) and Issinella ex gr. devonica (right). C-1(27), 10 - C-9[3/2(1)]. 

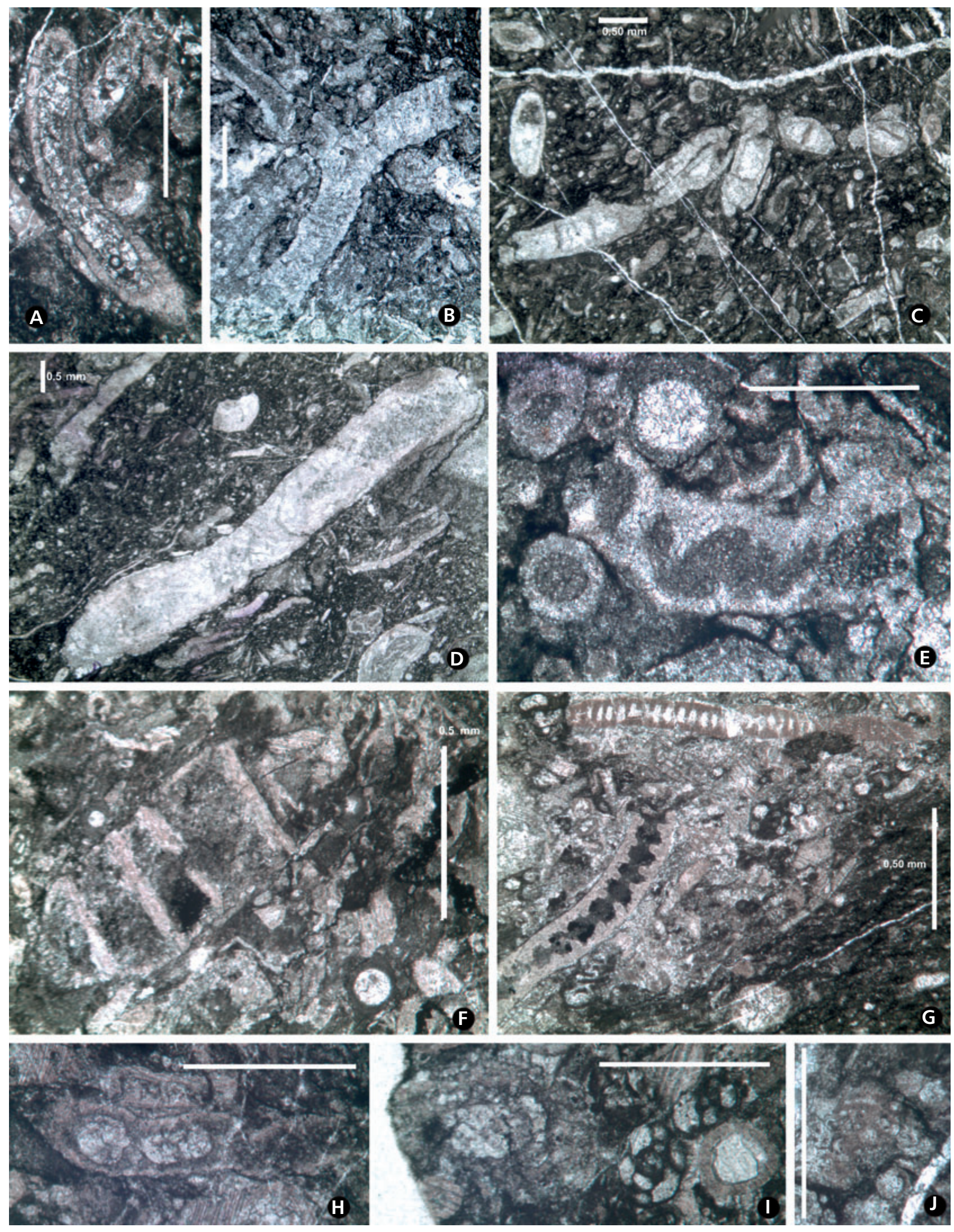
thin, clear, central layer, similar to that of Calcisphaera polydermoides (Conil \& Lys 1964). No apertures are obvious.

Material. - 52 specimens.

Repository of the types. - University Lille 1 collection (USTL1/1099).

Discussion. - Vicinesphaera squalida have a more homogeneously thicker wall of 0.020-0.060 mm (see Lipina 1955).

Occurrence. - MFZ11A-B of Cebeciköy.

Order Endothyrida Brady, nom. translat. Fursenko Superfamily Tournayelloidea Dain, nom. translat. Dain in Rauzer-Chernousova \& Fursenko, emend. Hance, Hou \& Vachard

Family Globoendothyridae Reitlinger in Voloshinova \& Reitlinger, nom. translat. Hance, Hou \& Vachard

Subfamily Globoendothyrinae Reitlinger in

Voloshinova \& Reitlinger

\section{Genus Planogloboendothyra Hance, Hou \& Vachard, 2011}

Planogloboendothyra modesta sp. nov. Figures 9P-R, 10A, 12T

?1980 Globoendothyra sp. - Conil et al., pl. 9, fig. 5.

?1982 Globoendothyra sp. 2. - Groessens et al., pl. 7, figs $150,151$.

Etymology. - Latin modestus, modest.

Holotype. - Fig. 10A, sample C-1.3(27).

Type horizon. - Late early Viséan (MFZ11A).

Type locality. - Cebeciköy (NW Turkey).

Diagnosis. - A species of Planogloboendothyra characterized by large test, compressed and biumbilicate; with few deviations of the coiling axis, except in the eventually excentred, last whorl.

Description. - The test is large with moderately numerous chambers, weakly oscillating coiling axes and asymmetrical semi-evolute last whorls, and broadly rounded peripheries. Proloculus spherical, relatively wide. There are up to 9 chambers in the last whorl, and they are well individualized although few inflated. Progressively enlarging, the successive chambers overlap partially the umbilical area, the test becoming nautiloid involute but remaining biumbilicate. In adult forms, the latest chambers are slightly inflated. In equatorial sections, the chambers appear subrectangular and vaulted whereas, in axial sections, they are oval, becoming kidney-shaped in the last whorls. Septa are moderately thick, slightly inclined forwards. The septa, as thick as the wall, straight, perpendicular to the spiral suture in equatorial section and curved, with a tangential approach to the spiral suture in more tangential sections. Sutures are prominent and incised. Supplementary deposits are represented by crustae and a terminal hook. The wall is relatively thick but moderately differentiated; it agglutinates scattered, poorly sorted fine-silt to silt-sized calcareous grains. The last whorl is deviated and slightly evolute. Aperture single basal.

Dimensions. $-\mathrm{D}=0.665-1.350 \mathrm{~mm}, \mathrm{w}=0.350-0.500 \mathrm{~mm}$, $\mathrm{w} / \mathrm{D}=0.37-0.52, \mathrm{n}=2.5-3$ (4.5) (with 7-9 chambers), $\mathrm{p}=0.030-0.080 \mathrm{~mm}, \mathrm{~h}=0.060-0.150(0.225) \mathrm{mm}, \mathrm{s}=$ $0.010-0.040(0.070) \mathrm{mm}$.

Comparisons. - It differs from Planogloboendothyra splendens Hance, Hou \& Vachard, 2011 by the nearly planispiral coiling and the larger dimensions $(\mathrm{D}=0.867$ to $1.000 \mathrm{~mm}, \mathrm{w}=0.373-0.467 \mathrm{~mm}, \mathrm{w} / \mathrm{D}=0.43-0.49$ for P. splendens); from $P$. arcuata (Grozdilova \& Lebedeva, 1954 ) by a larger test (although immature specimens are similar; Fig. 8R); from P. nekutchanica (Bogush, 1987), it differs by more deeper umbilici, less broad whorls, and a little larger dimensions $(\mathrm{D}=0.62-0.81 \mathrm{~mm}, \mathrm{w}=0.31-0.44 \mathrm{~mm}$ for $P$. nekutchanica); from $P$. insigna Postoyalko in Postoyalko \& Garan, 1972, which is also biumbilicate, it differs by less deviations of axis and a more compressed test, from P. ishimica (Rauzer-Chernousova, 1948), which has almost the same dimensions, by a test more compressed (w/D of $P$. ishimica $=0.69$ according to Grozdilova \& Lebedeva 1954, or 0.60-0.73 for Lebedeva 1954).

Material. - Ten sections.

Repository of the types. - University Lille 1 collection (USTL1/1100).

Figure 7. Scale bars $=0.5 \mathrm{~mm}$. $\bullet \mathrm{A}-\mathrm{C}-$ Issinella ex gr. devonica; A - associated with Earlandia ex gr. elegans and Endothyra paraprisca (bottom centre). C-1[2/2(2)], B - (left) with Palaeoberesella lahuseni (right). C-1.3(9), C - the perforations seem to be ramified (an exceptional character in this genus). C-8[3/2(3)]. - D-F - Issinella luteotubuliformis sp. nov.; D - detail of the holotype showing several dark diaphragms. C-9(6), E - paratype with a yellowish diaphragm. C-9(5), F - paratype, typical view of the individuals devoid of diaphragms. C-9(8). $\bullet \mathrm{G}-\mathrm{I}-$ Issinella enormis sp. nov.; G - three paratypes. C-11(5), H - a bifurcated paratype. C-11(1), I - three paratypes. C-11(3). 

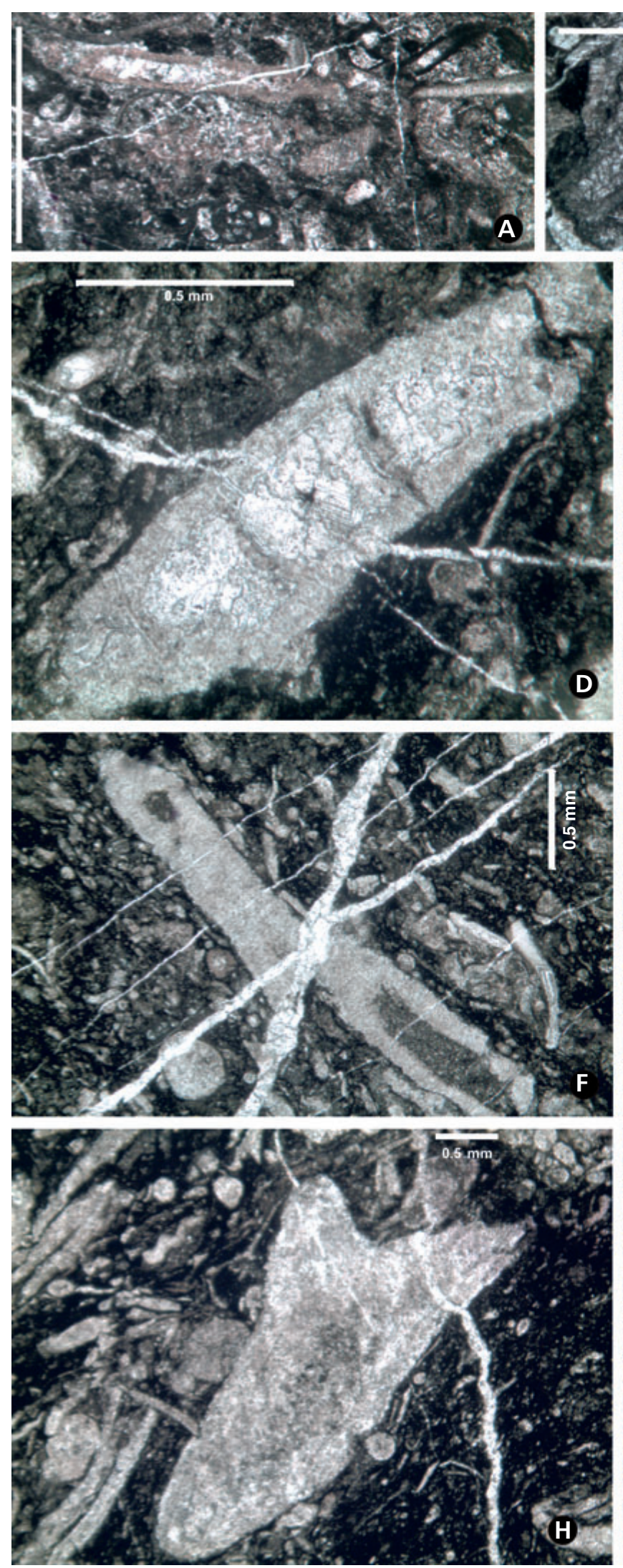
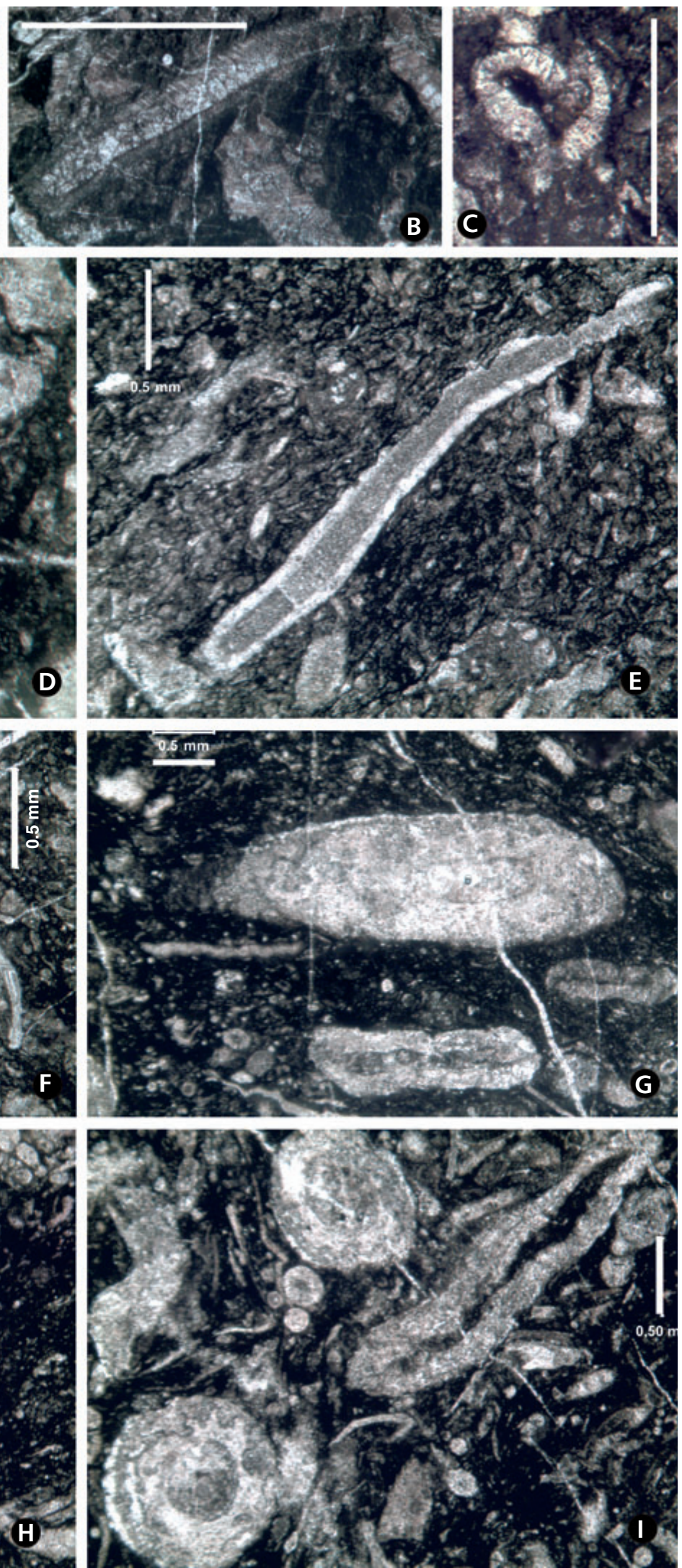
Occurrence. - Type locality and type horizon. Questionable in Belgium and England.

Family Endothyridae Brady, nom. translat. Rhumbler Subfamily Endothyrinae Brady

\section{Genus Endothyra Phillips sensu Brady, emend. China}

\section{Endothyra irinaeformis sp. nov.}

Figures 11A, J-N

Etymology. - Relatively similar to Endothyra irinae Reitlinger, 1950.

Holotype. - Fig. 11L, sample C-1(+2).

Type horizon. - Late early Viséan (MFZ11A).

Type locality. - Cebeciköy (NW Turkey).

Diagnosis. - A medium sized Endothyra very compressed (in axial as well as transverse planes), with very thick crustae, and a faintly differentiated wall.

Description. - Test free, lenticular, symmetric, slightly biumbilicate, with a rounded periphery. It is formed by a glo- bular, medium-sized proloculus followed by a succession of chambers, endothyroidally coiled on 2.5 to 3 whorls (the deviations of the whorls are generally approximately $60^{\circ}$ ). Slowly enlarging, the successive chambers overlap partially the umbilical area, the test becoming subdiscoidal involute. 8 chambers may be observed in the last whorl. Chambers hemispherical, typically endothyroid, increasing moderately in height. They communicate by a single, basal high median opening (probably an arch). In equatorial sections, the chambers appear subrectangular whereas, in axial sections, they are oval in the last whorls. The wall, microgranular, dominantly dark, is occasionally differentiated but less than in Mediendothyra. The septa, as thick as the wall, are straight, perpendicular to the spiral suture in equatorial section and slightly curved. The crustae are thick, with a curved forwards hook in the last chamber (Fig. 11L, N). The corner fillings are very developed (Fig. 11K) and occasionally resemble pseudochomata (Fig. 11M). Aperture single, basal, relatively low and narrow.

Dimensions. $-\mathrm{D}=0.550-0.700 \mathrm{~mm}, \mathrm{w}=0.170-0.240 \mathrm{~mm}$, $\mathrm{w} / \mathrm{D}=0.31-0.48, \mathrm{n}=2.5-3$ (for 8 chambers at last whorl), $\mathrm{p}=0.040 \mathrm{~mm}, \mathrm{~h}=0.100-0.150 \mathrm{~mm}, \mathrm{~s}=0.007 \mathrm{~mm}$.

Discussion. - It differs from E. irinae by more compressed transverse sections, more compressed and less evolute axial sections, and stronger crustae. It differs from E. apposita

Figure 8. Scale bar = $0.5 \mathrm{~mm}$ (see Fig. 8Y). - A, J-L? - Uralodiscus rotundus elongatus; A - C-1.2(3), J - C-9B(7), K - C-10.2(+1), L? - C-6(1). - B-D, F, L, N, O - Uralodiscus rotundus inflatus; B - C-1.3(21), C - C-1(2/2(3)), D - C-6(3), F - C-6(8), L - C-6(2), N - C-5[2/2(4)], O - C-5B(3). - E, G-I - Uralodiscus rotundus rotundus; E - C-6(6), G - C-8(5), H - C-8(6), I - C9B(1). - M? - deformed Uralodiscus sp. C-6.2(+2). $\bullet \mathrm{V}, \mathrm{W}-$ Uralodiscus rotundus vastus; $\mathrm{V}-\mathrm{C}-9(11), \mathrm{W}-\mathrm{C}-9(1) \cdot \bullet \mathrm{P}$ ?, Q-U - Paraarchaediscus (stage involutus) cf. sumsariensis; P? - C-5[2/2(1)], $\mathrm{Q}-\mathrm{C}-5[2 / 2(5)], \mathrm{R}-\mathrm{C}-5(+3), \mathrm{S}-\mathrm{C}-5[31 / 1(2)], \mathrm{T}-\mathrm{C}-5(+4 \mathrm{bis}), \mathrm{U}-\mathrm{C}-5[31 / 1(1)] \cdot \bullet \mathrm{X}, \mathrm{Y}-$ Conilidiscus cf. bucculentus; X - C-10(3), Y - C-9.1(10). $\cdot \mathrm{Z}$, AA, BB? - Paraarchaediscus (stage involutus) regularis; Z-C-10(5), AA-C-10(4), BB? -C-9.1(1). CC-Paraarchaediscus (stage concavus) aff. pauxillus. C-5.3(+1).

Figure 9. Scale bar $=0.5 \mathrm{~mm}$ (see Fig. 9E). $\bullet$ A - Kamaena pirleti. C-1.3(3). $\bullet \mathrm{B}-$ Palaeoberesella sp. 1. C-5B(6). C, D - Palaeoberesella lahuseni. Two axial sections with bracelets; C - C-10.2(6), D - C-10[10/2(4)]. - E - Exvotarisella index with Uralodiscus rotundus inflatus. C-1.3(4). $\bullet \mathrm{F}-$ Calcisphaera grandis (Lipina 1950). C-8(+1). $\bullet \mathrm{G}-$ Magnitella cf. agapovensis. C-10(8). $\bullet \mathrm{H}, \mathrm{J}, \mathrm{K}-$ Brunsia spirillinoides; $\mathrm{H}-$ with Exvotarisella index (top). C-1(19), J - with Vicinesphaera parasqualida sp. nov. (paratype; bottom, right). C-1(+5), K - with Vicinesphaera parasqualida sp. nov. (paratype), Endothyra prisca, Palaeoberesella lahuseni and Issinella ex gr. devonica. C-1.3(26). $\bullet$ I - Brunsia pulchra with Palaeoberesella lahuseni (top) and Issinella ex gr. devonica (bottom). C-1.3(20). $\bullet \mathrm{L}-\mathrm{O}-$ Forschia parvula; $\mathrm{L}-$ with Earlandia ex gr. minor, Exvotarisella index and Endothyra praesimilis. C-1(16), M - with Omphalotis aff. involuta. C-5B(1), N-C-5B(4), O - C-5. $\bullet$ P-R - Planogloboendothyra modesta sp. nov.; P - paratype with Vicinesphaera parasqualida sp. nov. (paratype, centre right). C-1(35), Q - paratype with Endothyra similis amplis (bottom, left) and Uralodiscus rotundus elongatus (top, right). C-1.3(40), R - paratype with Endothyra similis amplis (top, left and centre). C-1.3(38).

Figure 10. Scale bars $=0.5 \mathrm{~mm}$ (Fig. 10A, J have that of Fig. 10J; Fig. 10B-I, K-N, that of Fig. 10D). • A - Planogloboendothyra modesta sp. nov. Holotype. C-1.3(27). • B - Eotextularia diversa. C-8.4(2). • C - Viseina? sp. C-5[13/2(1)]. • D - Exvotarisella index. C-10.2(1). - E-G, J, M, N - Pseudolituotubella orientalis; E- C-1.2[13/2(1)], F- C-1.3(33), G - C-1(5), J - with Eotextularia diversa (top, right), Earlandia ex gr. minor (centre) and Endothyra paraprisca (top, left). C-6(9), M - C-7(1), N - with an Endothyra prisca. C-1(29). $\bullet$ H - Vicinesphaera parasqualida sp. nov. Holotype (left) and three paratypes (centre and right). C-1.2[13/2(4)]. $\bullet$ I - Baituganella ex gr. vulgaris. C-1.3(21). $\bullet$ - Magnitella cf. agapovensis. C-7[13/2(5)]. $\bullet$ L - Forschia parvula. C-7[13/2(3)]. 

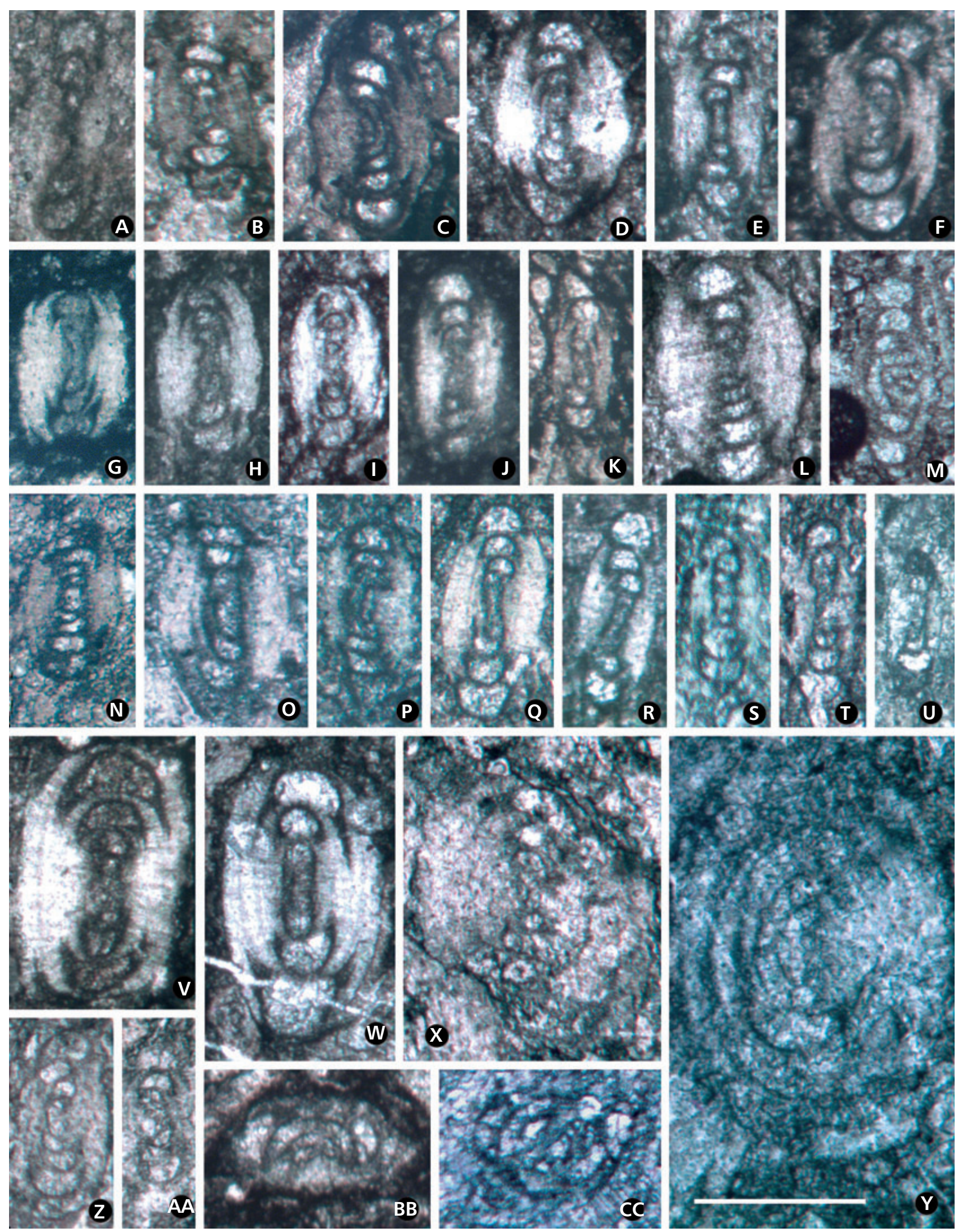

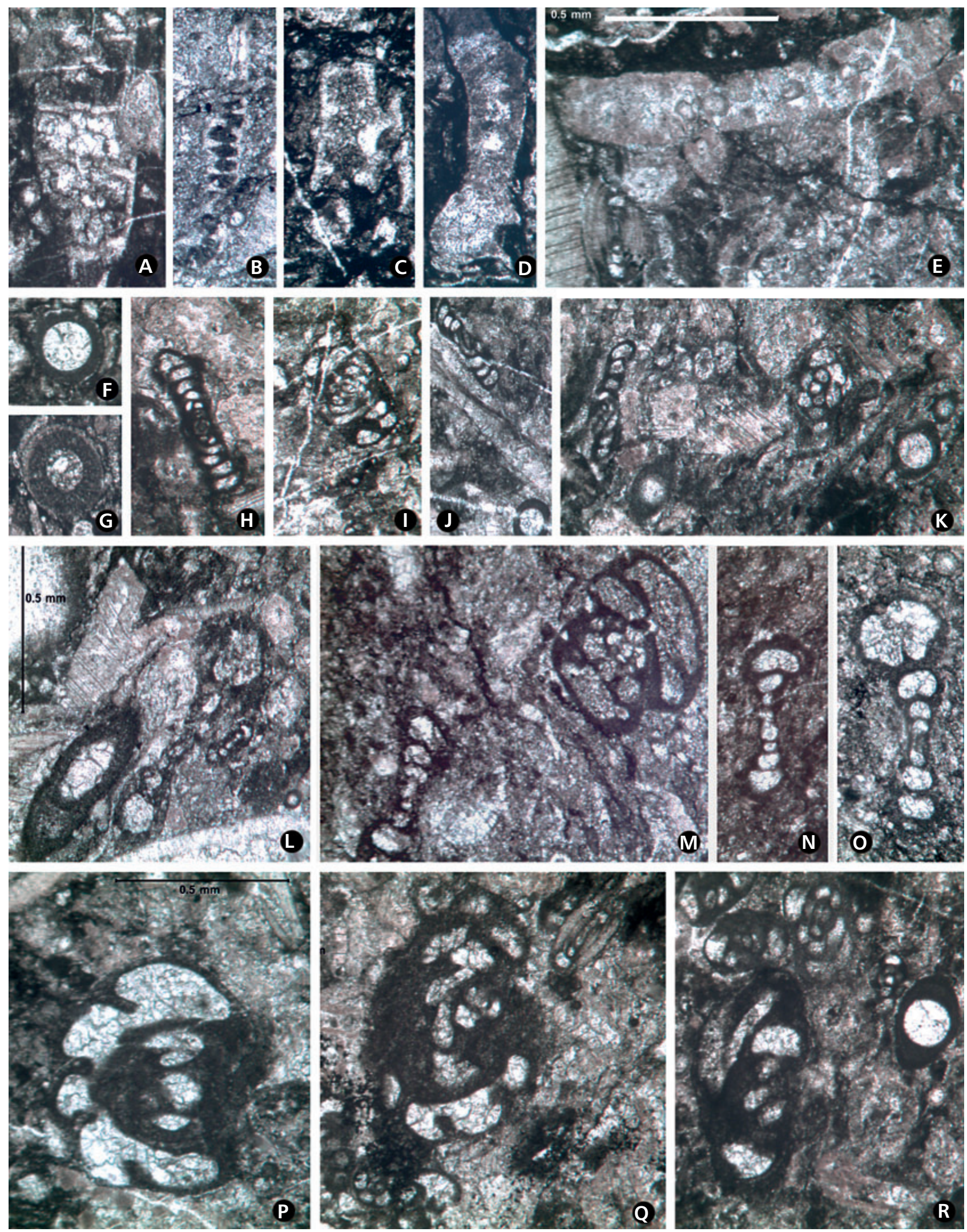

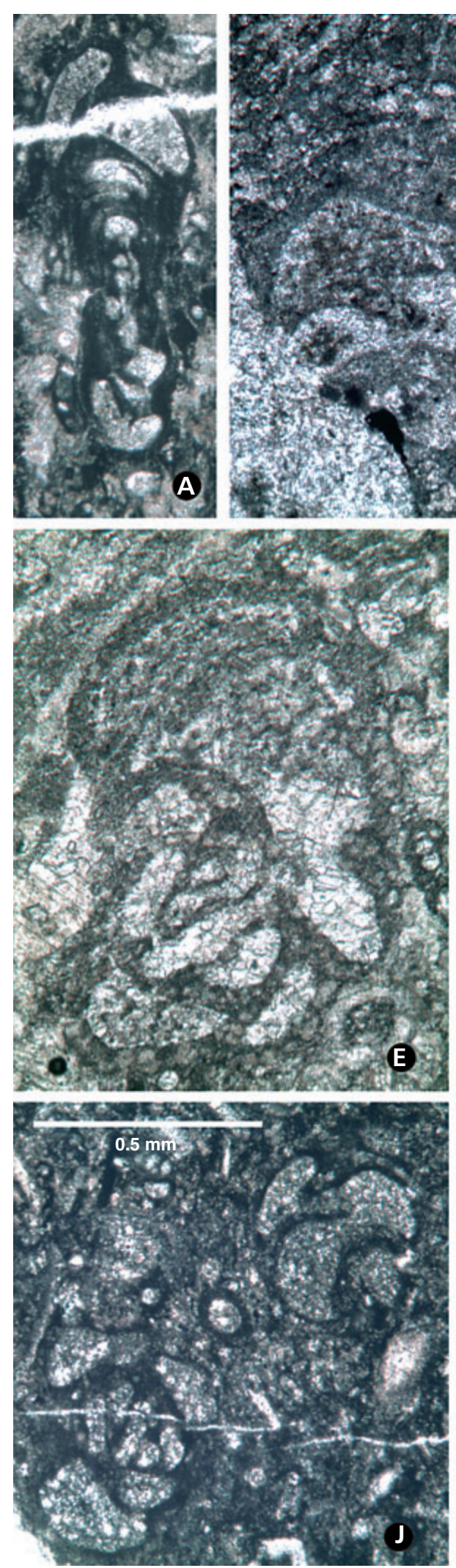
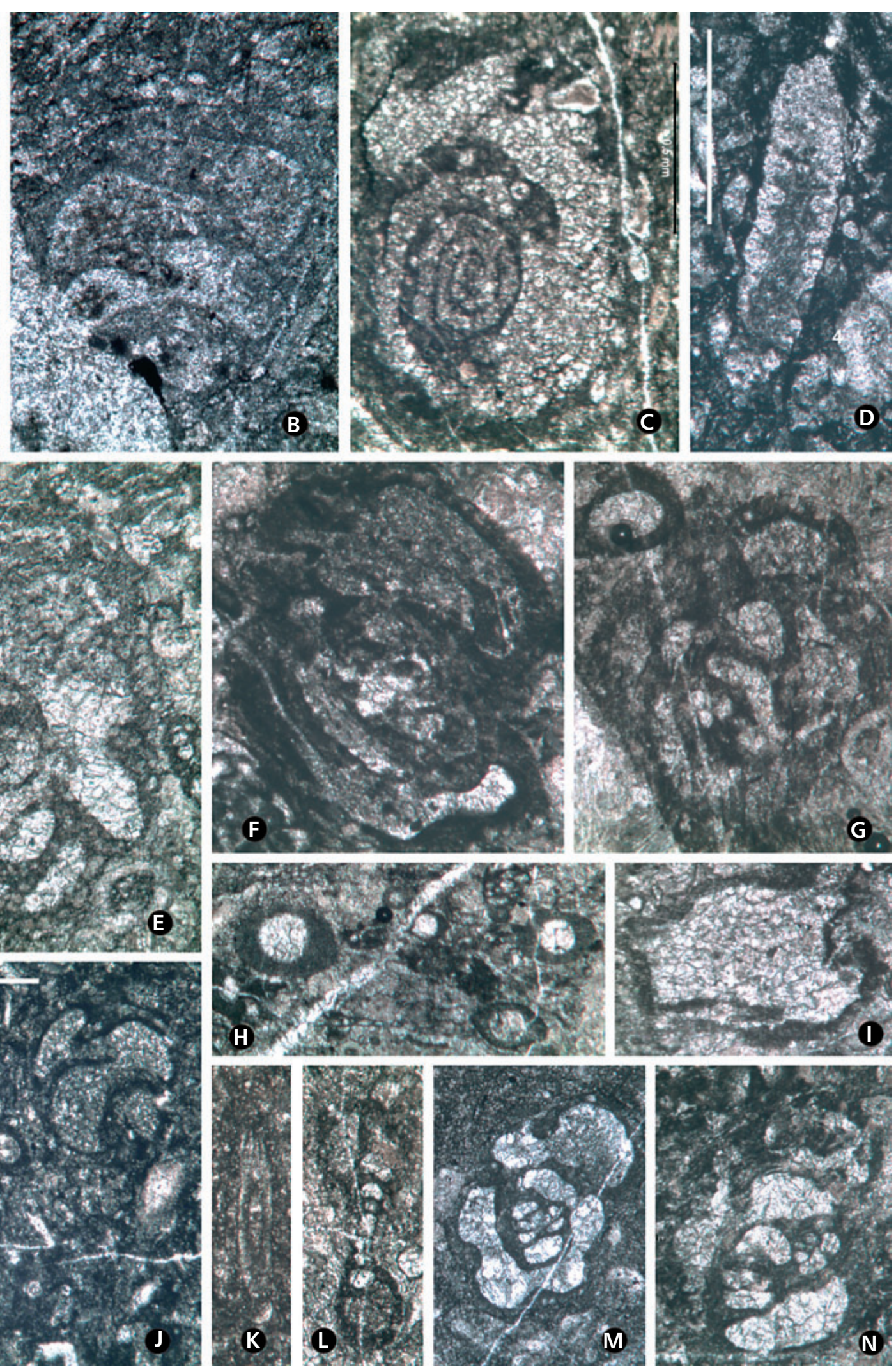
Ganelina, 1956 by the very more compressed test $(\mathrm{w} / \mathrm{D}=$ 0.61-0.67 in this latter species). It differs from E. neglecta Vachard, 1977 by the more compressed test and the very narrowly expanding spire. It differs from E. torta Conil \& Lys, 1968 by less deviations, less whorls and less chambers at the last whorl, but both species are probably phylogenetically related. From the more compressed Omphalotis and/or Mediendothyra, it remains distinct by more compressed and less differentiated walls.

Material. - Twelve sections.

Repository of the types. - University Lille 1 collection (USTL1/1101).

Occurrence. - Type locality and type horizon.

\section{Palaeogeographical hypothesis}

The Zonguldak and İstanbul terranes (Fig. 1) are often considered as one geodynamic unit (Okay \& Tüysüz 1999; Kalvoda 2003; Moix et al. 2008, 2011); nevertheless, as first admitted by Göncüoğlu \& Kozur (1998), they can be considered as distinct and included in some Viséan palaeogeographic models of western Europe (Franke \& Engel 1986, Korn 1997, Vachard et al. 2006, Pille 2008, Kalvoda et al. 2008) where the western Palaeotethys is subdivided in two branches (Fig. 14): a northern branch (partially linked in the Rheno-Hercynian and Saxo-Thuringian traditional German Zones or Brunovistulian Terrane: Franke \& Engel 1986, Yanev 2000, Kalvoda 2002, Weyer 2006, Meinhold et al. 2008, Kalvoda et al. 2008), and a southern branch (the Eovelebitella subprovince: Vachard et al. 2006 and Pille 2008; i.e., an extension of the Mediterranean Zone of Franke \& Engel 1986). Because of their complete lithological and microfaciological differences, both branches are probably separated by an emerged barrier (Fig. 14). Due to their common location in the subtropical or tropical domain during the Viséan, both branches have generally the same microfauna and microflora; nevertheless, a species like Quasiendothyra kobeitusana (Rauzer-Chernousova) seems only located in the northern branch. Although favourable carbonate facies existed in the southern branch
(Dil 1975, Vachard \& Clément 1994), the stenotherm and stenobath Quasiendothyra kobeitusana is absent because its migration is stopped by palaeotopographic or palaeoclimatic barriers. Eovelebitella Vachard is an excellent palaeobiogeographic index for the southern branch. We try to identify more segregating taxa.

The Zonguldak Terrane is probably located at the extremity of the northern branch (Fig. 13) because its Late Devonian, Mississippian and Early-Middle Pennsylvanian series are similar to those of the Dniepr-Donetz Basin and Dobrugea (Bulgaria), especially the foraminiferal succession composed of Quasiendothyra, Chernyshinella, Uralodiscus, Pojarkovella, Eostaffella, Euxinita, etc. (e.g., Vdovenko 1986). The algal microfauna remains poorly known.

The İstanbul Terrane is probably located at the extremity of the southern branch (Fig. 14). This terrane has many similarities with the Montagne Noire in southern France, at the western part of the same branch: Late Devonian red nodular limestone of griotte-type; Tournaisian black shales rich in radiolarian; rare MFZ10 olistoliths; first rich assemblages of foraminifers in MFZ11 olistoliths reworked in a flysch. Quasiendothyra kobeitusana is absent, but this palaeobiogeographic criterion is misleading, because of unfavourable lithofacies (radiolarian cherts or nodular micritic limestones). Similarly, carbonate deposits favourable to the presence of the shallow marine tropical Eovelebitella in the late Viséan deposits of the İstanbul Terrane were not encountered.

Southwards, the Turkish terranes are represented by the Sakarya composite terrane (Göncüoğlu et al. 1997; Figs 1, 14) or Sakarya complex (Y1lmaz 1991, Altıner \& Özgül 2001, Moix et al. 2008). The first name is preferred here because Sakarya is indeed a composite terrane with Variscan, Cimmerian and Alpine components, whereas the Sakarya complex refers only to the Karakaya unit, i.e., to the Palaeotethyan mélange. The Sakarya composite terrane is geodynamically connected with Chios, Rhodope, Pelagonia and Tizia (Meinhold et al. 2008, Okay et al. 2011). It is also eventually connected with the Alborz, due to the common and successive presence of the following foraminifers: Eoparastaffella simplex Vdovenko (Bozorgnia 1973, pl. 18, figs 6, 7, 13 and Leven \& Okay 1996, pl. 1, fig. 16: as Pseudoendothyra simplex), Pojarkovella

Figure 11. Scale bars $=0.5 \mathrm{~mm}$ (Fig. 11B-I, K-T have that of Fig. 10T). • A - Pseudolituotubella aff. tschernyshevi (bottom, right) with Plectogyranopsis convexa (top, left) and Endothyra irinaeformis sp. nov. (paratype; top, centre). C-1.3(31). • B-D - Condrustella cf. modavensis; B - C-5(2), C - C-6(6), D - C-7[13/3(1)]. • E, F - Eotextularia cf. mongeri; E - C-1(30), F - C-1(4). • G, H, Q - Endothyra similis inops, G - with Vicinesphaera parasqualida sp. nov. (paratype, top right), C-1(+5), H - C-1(5), Q - C-7[3/2(2)]. • I - Endothyra praesimilis. C-5(+4bis). - J-N - Endothyra irinaeformis sp. nov.; J - paratype (top left) with Endothyra prisca (top, centre), Earlandia ex gr. minor (top, right), Endothyra similis inops (bottom, left) and Mediocris? sp. (bottom, right). C-1(2), $\mathrm{K}$ - paratype (top, centre) with Inflatoendothyra? sp. (bottom, left) and Endothyra similis inops (centre, right). C-1, L - holotype (left) with Earlandia ex gr. elegans (right). C-1(+2), M - paratype (left) with Endothyra similis amplis (top, right). C-1.3(39), N - paratype. C-1.3(42). • O - Endospiroplectammina cf. conili. C-1[2/2(5)]. • P - Mediendothyra barzassiensis. C-1[10.2(3)]. - R - Endothyra acantha. C-7[3/2(2)]. S, T - Endothyra similis amplis, S - C-1(+3), T - C-1.2 [13/2(2)]. 

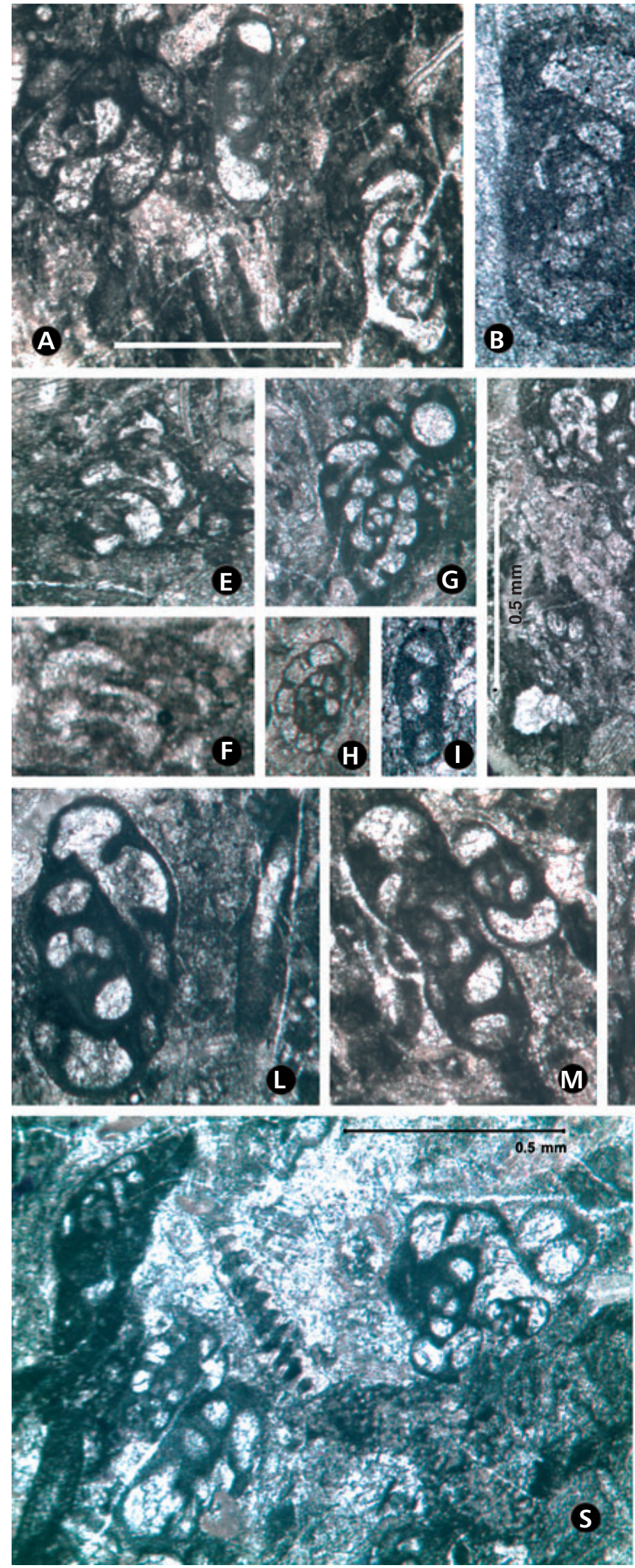
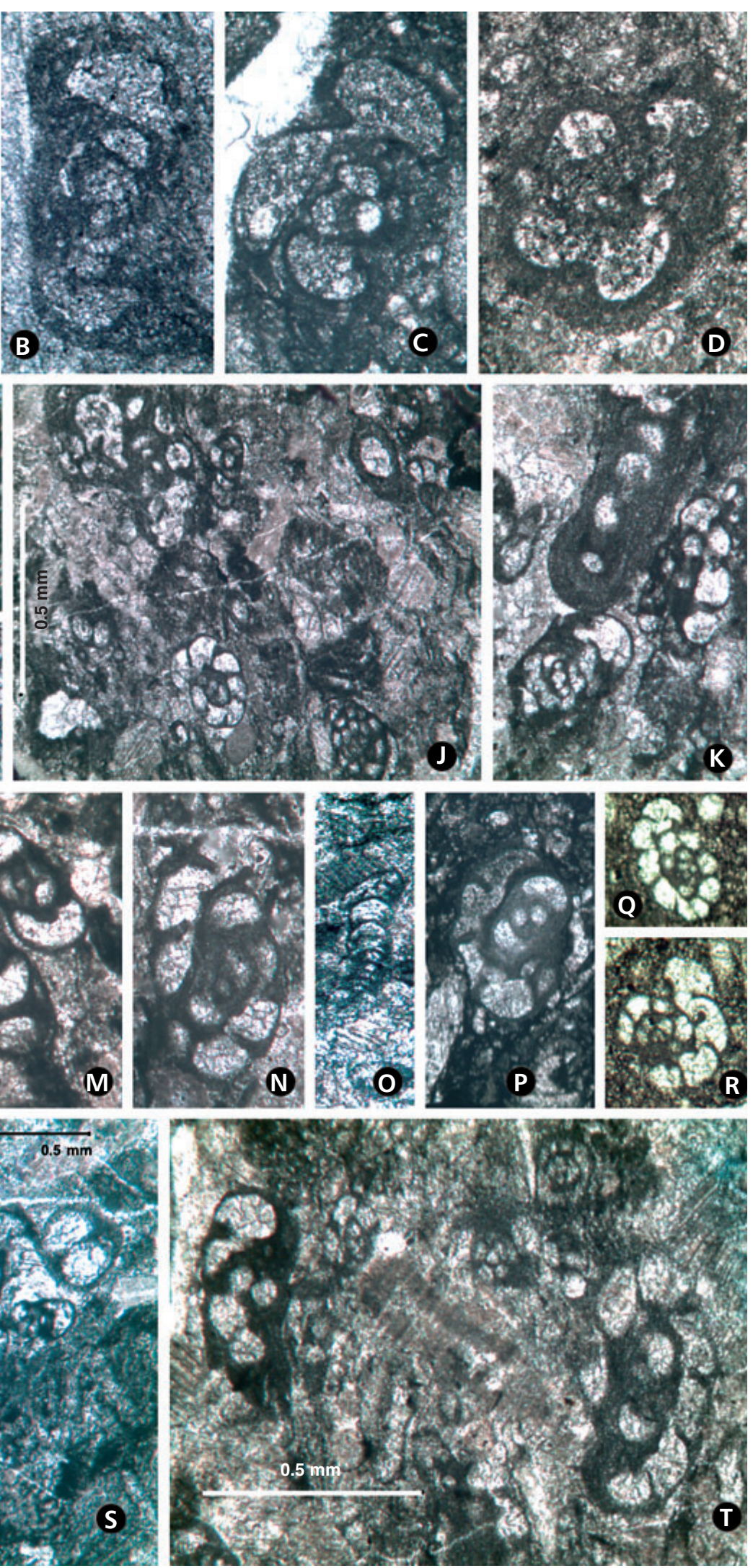
ex gr. nibelis (Zandkarimy \& Vachard, unpublished data and Leven \& Okay 1996, pl. 1, fig. 26: as Endothyranopsis sp.), and Howchinia gibba (Möller) (Bozorgnia 1973, pl. 29, figs 5, 7, 8 and Leven \& Okay 1996, pl. 1, fig. 15).

Due to this probable location and composition of its foraminiferal assemblages, the İstanbul Terrane seems to be located at a palaeobiogeographical crossroads or under several currents crossing these areas. Consequently, it received rapidly the new genera and species from the centres of radiation of Siberia, Kazakhstan, and adjacent southern Urals, and dispatched them to (a) Sakarya and Alborz, (b) Taurus and Egypt; (c) westernmost parts of the southern branch of Palaeotethys. Nevertheless, the very great rarity of the loeblichioids in the Cebeciköy Limestone strongly constrasts with the diversity of this superfamily in the Alborz (Bozorgnia 1973, Devuyst 2006, Hance et al. 2011).

\section{Conclusions}

1. The Cebeciköy Limestone, which covers vast areas on both sides of the Bosphorus in the İstanbul Terrane (NW Turkey), yielded new micropalaeontology data.

2. The cyanobacteria are very rare; true green and red algae absent; moravamminids (incertae sedis algae algospongia), always frequent, show two new species: Issinella luteotubulifomis sp. nov. and I. enormis sp. nov.; aoujgaliids are rare or very rare.

3. The foraminifers are always present but more or less diversified and more or less abundant; some groups are entirely absent: dainellids, loeblichiids, true Globoendothyra or Eogloboendothyra, and Valvulinella. It is a mixed heterozoan-photozoan assemblage developed in a subtropical to warm temperate climatic zone ( $30-40^{\circ}$ of latitude).

4. Interesting taxa (yet described) in Cebeciköy are: Uralodiscus rotundus, Conilidiscus spp., Paraarchaediscus (stage involutus) sumsariensis-minimus, Magnitella spp., Pseudolituotubella orientalis, Condrustella modavensis, Eotextularia diversa, E. mongeri, Mediendothyra barzassiensis, Latiendothyranopsis spp., and Eoendothyranopsis spp.

5. Three species are new: Vicinesphaera parasqualida sp. nov., Planogloboendothyra modesta sp. nov., and Endothyra irinaeformis sp. nov.

6. The deposits correspond to the biozone MFZ11. Our data in Turkey confirm the data in Belgium and South China and permit to confirm and precise the boundary of the two subzones MFZ11A and MFZ11B.

7. The boundary between both subzones is characterized by the FAD of Conilidiscus and Paraarchaediscus and LAD of Eoendothyranopsis.

8. The palaeobiogeographical value of some taxa [e.g., Uralodiscus rotundus, Conilidiscus spp., Paraarchaediscus (stage involutus) sumsariensis-minimus] permits to provide some precisions about the reciprocal location of the Turkey terrane and their relationships with the adjacent areas.

9. The Zonguldak Terrane can be connected to the northern branch of the Palaeotethys and İstanbul Terrane belongs to the southern branch of this ocean.

10. The İstanbul Terrane seems to be located at a palaeobiogeographical crossroads which received the new taxa of the centres of radiation of southern Urals, Kazakhstan and Siberia, and dispatched them to (a) western Palaeotethys, (b) Sakarya and Alborz, (c) Taurus and Egypt.

Figure 12. Scale bars $=0.5 \mathrm{~mm}$ (Fig. 12A-S, U have that of Fig. 11P). A-C - Mediendothyra barzassiensis; A $-\mathrm{C}-1(13), \mathrm{B}-\mathrm{C}-1(1), \mathrm{C}-\mathrm{C}-6(2 \mathrm{bis})$. - D-F - Omphalotis aff. involuta; D - C-1.2(+2), E - C-1.3(20), F- C-1.3(43). $\bullet \mathrm{G}-$ Endothyra gratiodentalis. C-7[13/2(4)]. $\bullet$ H-J - Omphalotis cf. parasamarica; $\mathrm{H}$ - with Earlandia ex gr. minor (top, left), Endothyra prisca (centre) and E. similis amplis (top, right). C-1.2[13/2(3)], I - with Uralodiscus rotundus elongatus. C-10.2(+1), $\mathrm{J}-\mathrm{C}-8[3 / 2(3)] . \cdot \mathrm{K}-$ Eotuberitina reitlingerae. C-5(+3). $\bullet \mathrm{L}-$ Endothyra similis amplis. C-6(5). - M, O - Latiendothyranopsis? cf. menneri; M - C-6(5bis), O - C-6(1). • N - Endothyra praesimilis with Girvanella wetheredii. C-5[2/2(2)]. $\cdot \mathrm{P}-$ Plectogyranopsis ampla with two Brunsia spirillinoides. C-1.3(28). $\bullet \mathrm{Q}-$ Plectogyranopsis cf. dendrei. C-7[3/2(3)]. $\bullet \mathrm{R}-$ Plectogyranopsis convexa. C-1(4). $\bullet \mathrm{S}-$ Endothyra similis inops. C-1(4). $\bullet \mathrm{T}-$ Eoendothyranopsis $\mathrm{cf}$. ermakiensis (top, left) with Planogloboendothyra modesta sp. nov. (paratype; top, right), Issinella ex gr. devonica (bottom left and right), Palaeoberesella lahuseni (centre, left) and Earlandia ex gr. elegans (top, left). C-1(17). - U - Eoendothyranopsis donica tumefacta, with Endospiroplectammina cf. conili, Vicinesphaera parasqualida sp. nov. (paratype), Palaeoberesella lahuseni, and Earlandia ex gr. elegans. C-1(33).

Figure 13. Scale bars =0.5 mm (Fig. 13A, D-P have that of Fig. 13M). - A, E - Eoendothyranopsis donica tumefacta; A - C-1.2(1), E - C-1(25). - B-D - Eoendothyranopsis cf. ermakiensis; B - with Issinella ex gr. devonica (centre), Endothyra praesimilis (top, left), and E. paraprisca (bottom, centre). C-1.3(35), C-C-1.2(2), D - with Palaeoberesella lahuseni (bottom, right), Endothyra praesimilis (top, right) and with Vicinesphaera parasqualida sp. nov. (paratype, top right), C-1(34). • F, G - Tetrataxis immatura; F - C-6(1), G - C-1(28). • H, J - Tetrataxis aff. angusta; H - C-6(1bis), $\mathrm{J}-\mathrm{C}-5[31 / 1(3)] \cdot \bullet \mathrm{I}, \mathrm{M}-$ Tetrataxis bogushi $\mathrm{I}-\mathrm{C}-5 \mathrm{~B}(5), \mathrm{M}-$ Globobotetrataxis stage. C-5(3). $\bullet \mathrm{K}, \mathrm{L}-$ Tetrataxis cf. quasiconica; K - with Uralodiscus rotundus vastus. C-10.2(2), L - C-1.3(13). $\bullet \mathrm{N}-$ Mediocris breviscula. $\mathrm{C}-1(3) \cdot \bullet \mathrm{O}, \mathrm{P}$, , Q, R, T? - Eoparastaffella simplex lata; O - C-1.3(22), $\mathrm{P}$ ? - with Uralodiscus rotundus rotundus. C-6.2(+1), Q - C-8(4), R-C-8(1), T? - C-10(2). - S, U-X - Eoparastaffella simplex ovalis; S - C-1(5), U - C-1.2(5), V - C-1(10), W - C-1(+6), X-C-10(9). 

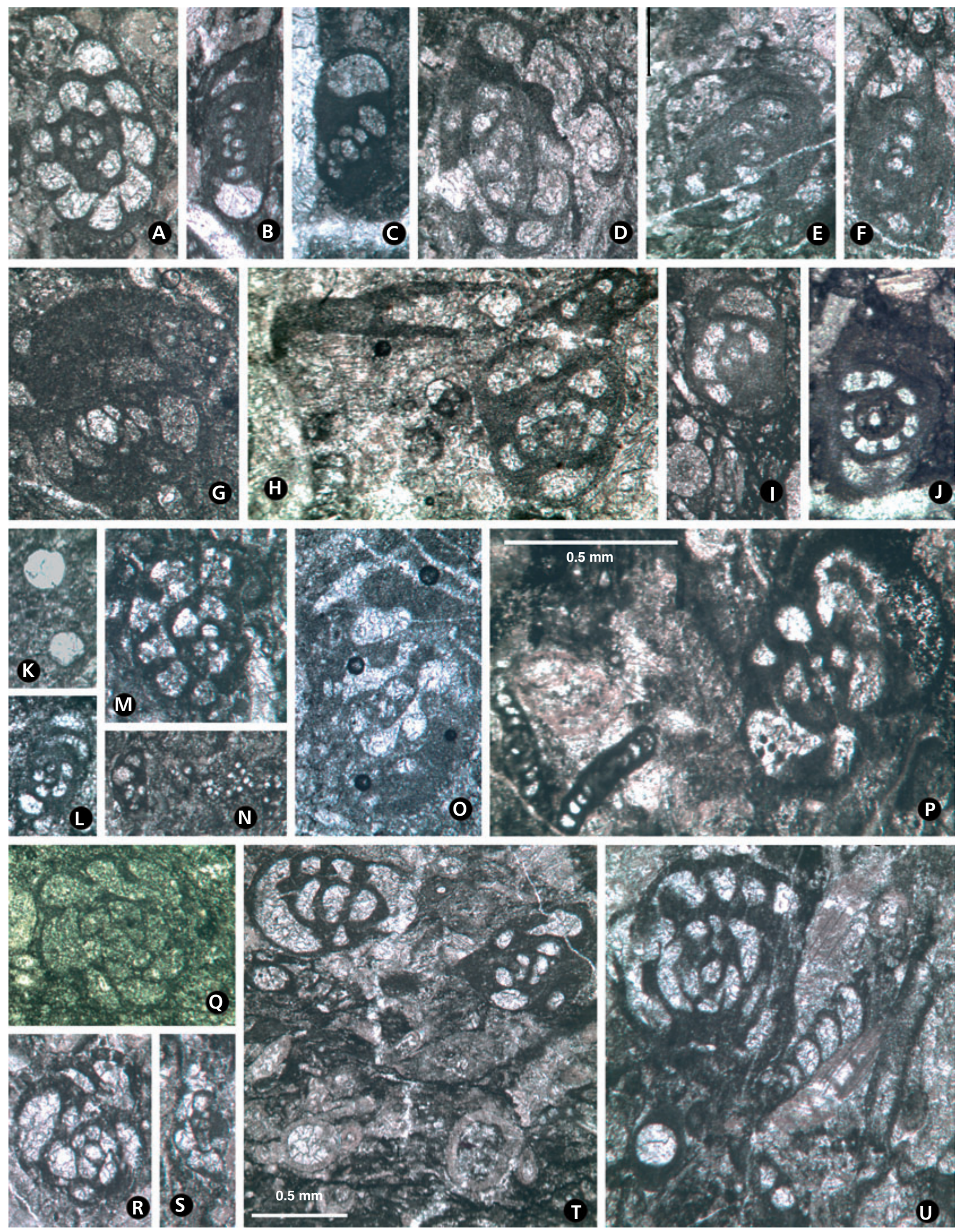

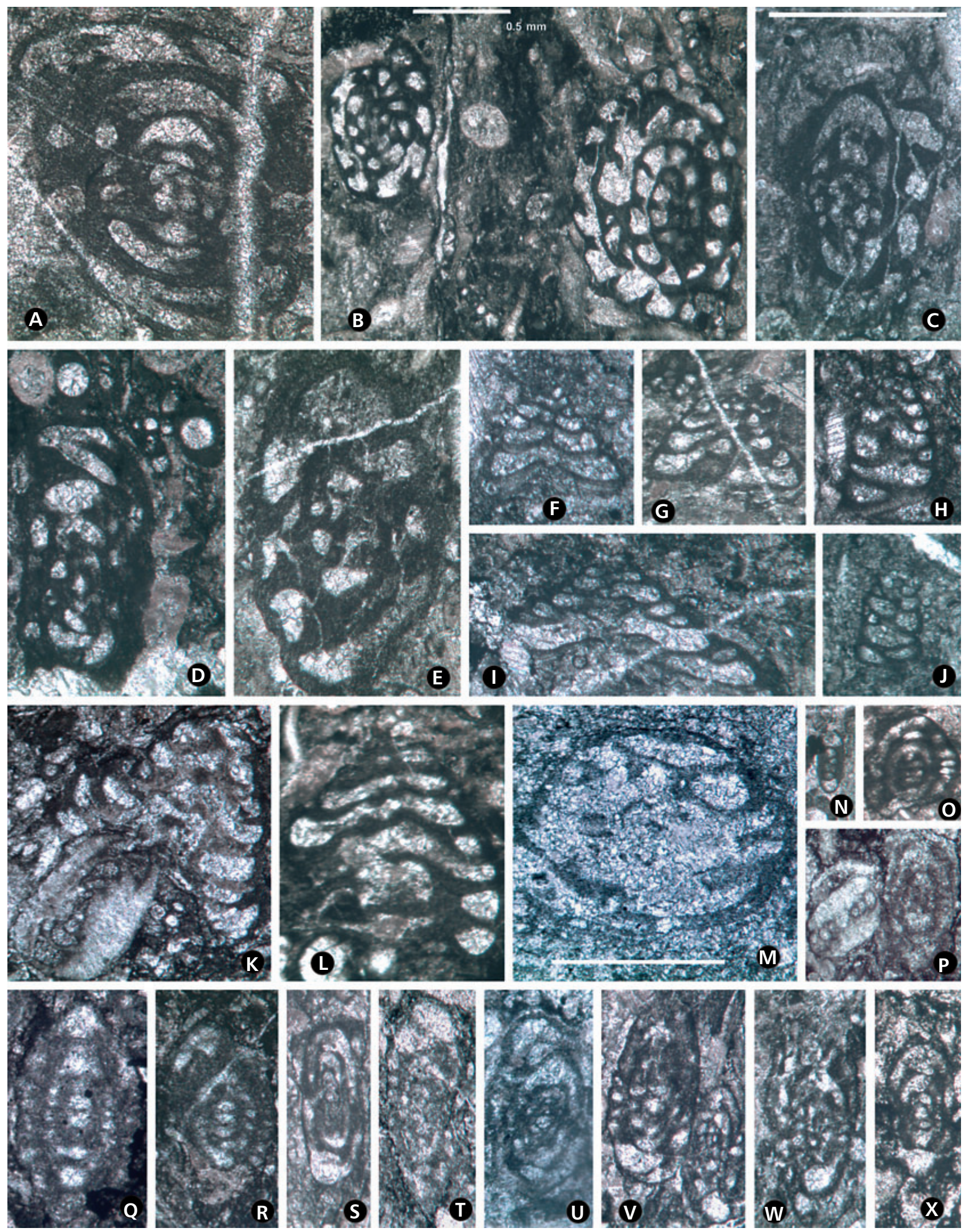


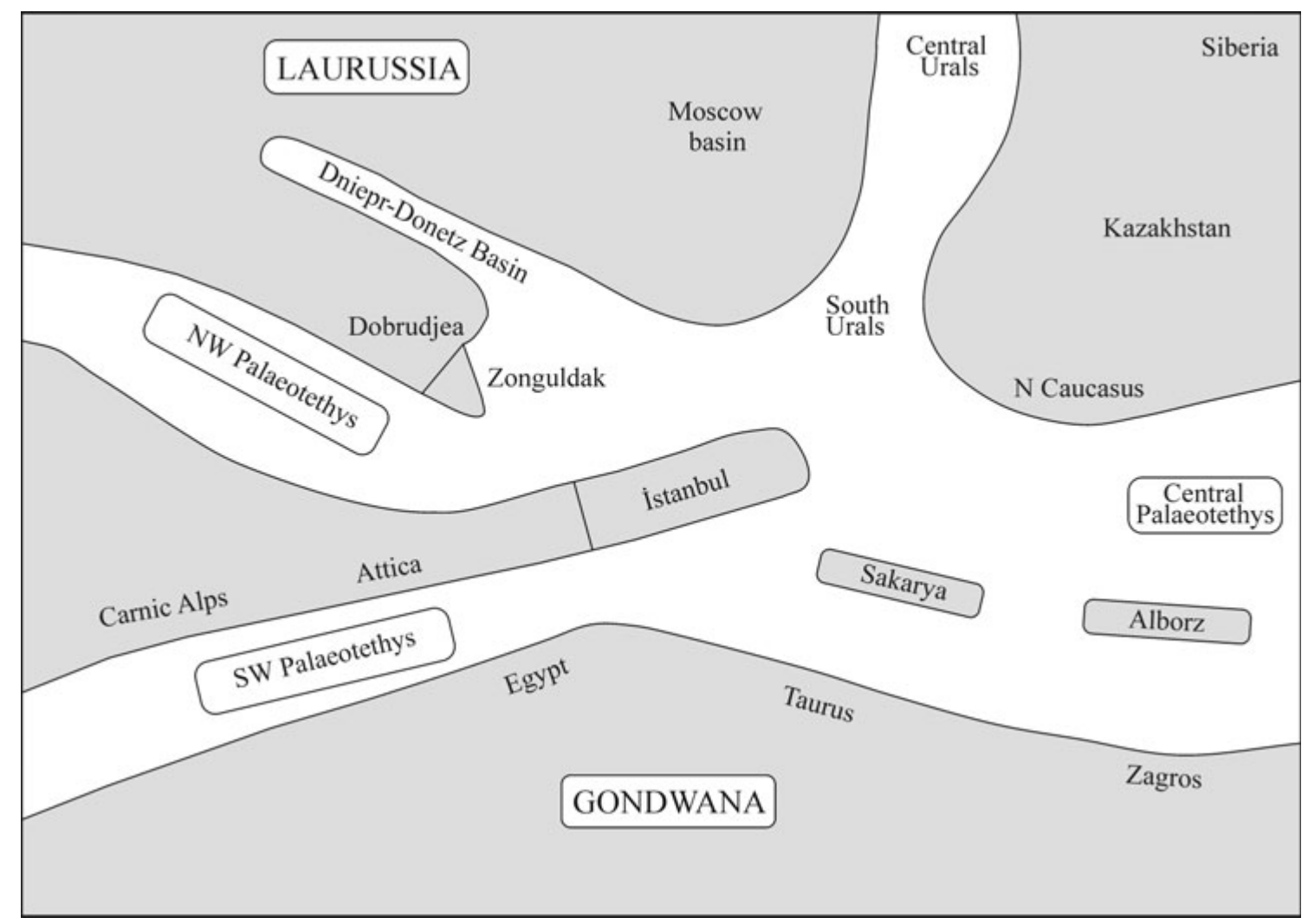

Figure 14. Palaeogeographic reconstruction of the mainlands and islands in western and central Palaeotethys based on foraminiferal palaeobiogeographic data.

\section{Acknowledgements}

This paper is a product of a joint-project (102Y157) between BAS (Bulgaria) and TUBITAK (Turkey). The authors acknowledge both institutions and MTA (Turkey) for their financial support. They are also grateful to the project members N. Özgül and İ. Gedik for their contributions in the field work. Thanks to Sylvie Grard, Sébastien Clausen, Deborah Ponlevé, Jessie Cuvelier, Léa Devaere (Villeneuve d'Ascq) and Thérèse Vachard (Gruson) for their technical help. Thanks to the constructive remarks of the reviewers: Jiří Kalvoda, Ian Somerville and Eddy Poty.

\section{References}

AltineR, D. 1981. Recherches stratigraphiques et micropaléontologiques dans le Taurus Oriental au NW de Pinarbași (Turquie). Thesis, University of Geneva.

Altiner, D. \& ÖzGÜL, N. 2001. Carboniferous and Permian of the allochthonous terranes of the Central Tauride Belt, Southern Turkey. PaleoForams 2001, International Conference of Paleozoic Benthic Foraminifera, Ankara, Guidebook, 35 pp.

ANTROPOV, I.A. 1950. New foraminiferal species of the Late Devonian of some areas of the eastern Russian Platform. Izvestiya Kazanskogo filiala, Geologicheskiy institut 1, 21-33. [in Russian]
Bogush, O.I. 1987. Systematics of the genus Globoendothyra (foraminifers). In Elkin, E.A. \& Yadrenkina, A.G. (eds) Microfauna and biostratigraphy of the Phanerozoic of Siberia. Nauka, Novosibirsk. [in Russian]

Bogush, O.I., Ivanova, R.M. \& Luchinina, V.A. 1990. Calcareous algae from the late Famennian and Early Carboniferous of Urals and Siberia. Trudy Instituta Geologii i Geofiziki 745, 1-160. [in Russian]

Bozorgnia, F. 1973. Paleozoic foraminiferal biostratigraphy of central and east Alborz Mountains, Iran. 185 pp. National Iranian Oil Company, Geological Laboratories, Publication 4, Tehran.

Brenckle, P.L. 2004. Late Visean (Mississippian) calcareous microfossils from the Tarim Basin of western China. Journal of Foraminiferal Research 34(2), 144-164. DOI 10.2113/0340144

Brenckle, P., Gaetani, M., Angiolini, L. \& Bahrammanesh, M. 2009. Refinements in biostratigraphy, chronostratigraphy, and paleogeography of the Mississippian (Lower Carboniferous) Mobarak Formation, Alborz, Iran. GeoArabia 14(3), 43-78.

Caridroit, M., Degardin, J.M., Derycke, C., Lethiers, F., Marcoux, J., Milhau, B., Pillevuit, A. \& Vachard, D. 1997. Un assemblage microfaunistique remarquable du Paléozoïque supérieur de Turquie (radiolaires, conodontes, ostracodes, foraminifères, microrestes de vertébrés). Géobios, Mémoire spécial 20, 109-115. DOI 10.1016/S0016-6995(97)80016-2

Çatal, E., Demirtasli, E., Dil, N., Kaya, O., Kiragli, C. \& 
Salanci, A. 1978. Field Excursions on the Carboniferous Stratigraphy in Turkey. Guidebook, IUGS Subcommission on Carboniferous Stratigraphy, 45 pp. Ankara.

Chuvashov, B.I. 1965. Foraminifers and algae of the Late Devonian deposits from the western slope of central and southern Urals. Devonian and Permian Foraminifera of the Urals. Sbornik po stratigrafii 8(74), 3-154. Institute of Geology, Ural Branch of the USSR, Sverdlovsk. [in Russian]

Conil, R. \& Groessens, E. 1974. Excursion B. International Symposium on Belgian micropaleontological limits, Namur 1974, 1-26.

Conil, R., Groessens, E., Laloux, M. \& Poty, E. 1989. La limite Tournaisien/Viséen dans la région-type. Annales de la Société Géologique de Belgique 112(1), 177-189.

Conil, R., Groessens, E., Laloux, M., Poty, E. \& Tourneur, F. 1991. Carboniferous guide foraminifera, corals and conodonts in the Franco-Belgian and Campine basins; their potential for widespread correlations. Courier Forschungsinstitut Senckenberg 130, 15-30.

Conil, R., Groessens, E. \& Pirlet, H. 1977. Nouvelle charte stratigraphique du Dinantien type de la Belgique. Annales de la Société géologique du Nord 96, 363-371.

Conil, R., Longerstaey, P.J. \& Ramsbottom, W.H.C. 1980. Matériaux pour l'étude micropaléontologique du Dinantien de Grande-Bretagne. Mémoires de l'Institut Géologique de l'Université de Louvain 30 (dated 1979), 1-115.

Conil, R. \& Lys, M. 1964. Matériaux pour l'étude micropaléontologique du Dinantien de la Belgique et de la France (Avesnois). Pt. 1, Algues et foraminifères; Pt. 2, Foraminifères (suite). Mémoires de l'Institut de Géologie de l'Université de Louvain 23, 1-372.

ConIL, R. \& Lys, M. 1968. Utilisation stratigraphique des foraminifères du Dinantien. Annales de la Société géologique de Belgique 91, 491-558.

Conil, R. \& Naum, C. 1976. Les foraminifères du Viséen moyen V2a aux environs de Dinant. Annales de la Société géologique de Belgique 99, 109-142.

Davydov, V.I., Crowley, J.L., Schmitz, M. D. \& Poletaev, V.I. 2010. High-precision U-Pb zircon age calibration of the global Carboniferous time scale and Milankovitch band cyclicity in the Donets Basin, eastern Ukraine. Geochemistry, Geophysics, Geosystems 11(1), 1-22.

Devuyst, F.-X. 2006. The Tournaisian-Viséan boundary in Eurasia. Definition, biostratigraphy, sedimentology and early evolution of the genus Eoparastaffella (foraminifer). $\mathrm{PhD}$ thesis, Catholic University of Louvain.

DiL, N. 1975. Etude micropaléontologique du Dinantien de Gokgöl et Kokaksu (Turquie). Annales de la Société géologique de Belgique 98, 213-228.

DiL, N. 1977. Assemblages caractéristiques de Foraminifères du Dévonien supérieur et du Dinantien de Turquie (Bassin carbonifère de Zonguldak). Annales de la Société Géologique de Belgique 99(2), 373-400.

Dil, N., Termier, G., Termier, H. \& Vachard, D. 1977. Contribution à l'étude stratigraphique et paléontologique du Viséen supérieur et du Namurien inférieur du bassin houiller de Zonguldak (Nord-Ouest de la Turquie). Annales de la Société géologique de Belgique 99(2), 401-449.

DoJen, C., ÖZGÜL, N., GöNCÜOĞLU, Y. \& GöNCÜOĞLU, M.C. 2004.
Early Devonian ostracods of Thuringian ecotype from NW Anatolia. Neues Jahrbuch für Geologie und Paläontologie, Monatshefte 12, 733-748.

EINOR, O.L. 1996. The former USSR, 13-407. In WAGNER, R., WinkLer Prins, C.F. \& Granados, L.F. (eds) The Carboniferous of the World, Instituto Tecnológico GeoMinero de España (IGME) \& Nationaal Natuurhistorisch Museum 3.

FRANKE, W. \& ENGEL, W. 1986. Synorogenic sedimentation in the Variscan Belt of Europe. Bulletin de la Société géologique de France 8, 2(1), 25-33.

GALLAGHER, S.J. 1998. Controls on the distribution of calcareous foraminifera in the Lower Carboniferous of Ireland. Marine Micropaleontology 34, 187-211.

DOI 10.1016/S0377-8398(98)00006-1

GanelinA, R.A. 1956. Visean foraminifers from the northwestern areas of the Submoscow Depression. Trudy VNIGRI 98, Mikrofauna SSSR 8, 61-159. [in Russian; French translation: BRGM 1626, 1-81.]

GönCÜOĞLU, M.C., Boncheva, I. \& GöncüOĞLU, Y. 2004. First discovery of middle Tournaisian conodonts in the griotte-type nodular pelagic limestones, İstanbul area, NW Turkey. Rivista Italiana di Paleontologica e Stratigrafia 110(2), 431-439.

GöncüoĞlu, M.C., ÇaPkinoĞLu, S., Gürsu, S., Noble, P., Turhan, N., Tekin, U.K., OKuyucu, C. \& GöncüoĞLu, Y. 2007. The Mississippian in the Central and Eastern Taurides (Turkey): constraints on the tectonic setting of the TaurideAnatolide Platform. Geologica Carpathica 58, 427-442.

GöNCÜOĞLu, M.C., DiRIK, K. \& KozLu, Y. 1997. General characteristics of pre-Alpine and Alpine Terranes in Turkey: explanatory notes to the terrane map of Turkey. Annales géologiques des pays helléniques $37,515-536$.

GöNCÜOĞLU, M.C. \& KozUR, H. 1998. Remarks on the preVariscan development in Turkey. In Linnemann, U., Heuse, T., Fatka, O., KRaft, P., Brocke, R. \& Erdtmann, B.T. (eds) Pre-Variscan Terrane Analysis of "Gondwanan Europe", Proceedings, Schriften des Staatlichen Museums Mineralogie und Geologie Dresden 9.

GÖrür, N., Monod, O., OKAY, A.I., ȘEngör, A.M.C., TÜysüZ, O., YiĞITBAS, E., SAKINÇ, M. \& AKKÖK, R. 1997. Paleogeographic and tectonic position of the Carboniferous rocks of the western Pontides (Turkey) in the frame of the Variscan belt. Bulletin de la Société géologique de France 168(2), 197-205.

Groessens, E., Conil, R. \& Hennebert, M. 1982. Le Dinantien du sondage de Saint Ghislain, stratigraphie et paléontologie. Mémoires pour servir à l'explication des cartes géologiques et minières de la Belgique, Mémoire 22, 1-137.

Grozdilova, L.P. \& Lebedeva, N.S. 1954. Foraminifers of the Early Carboniferous and Bashkirian stage of the Middle Carboniferous of the Kolvo-Vishera Basin. Trudy VNIGRI 81, Mikrofauna SSSR 7, 4-203. [in Russian]

Güvenç, T. 1965. Etude stratigraphique et micropaléontologique du Carbonifère et du Permien des Taurus occidentaux dans l'arrière-pays d'Alanya (Turquie). $\mathrm{PhD}$ thesis, University of Paris.

HAAs, W. 1968. Das Alt-Paläozoikum von Bithynien (Nordwest Türkei). Neues Jahrbuch für Geologie und Paläontologie, Abhandlungen 131, 178-242.

Hance, L. 1988. Le Moliniacien du Synclinorium de Dinant (Belgique), de la région dinantaise à la vallée de l'Ourthe. 
Mémoires de l'Institut Géologique de l'Université de Louvain 33, 1-91.

HANCE, L., Hou, H.F. \& VAchaRd, D. 2011. Upper Famennian to Viséan foraminifers and some carbonate microproblematica from South China, Hunan, Guangxi and Guizhou. 359 pp. Geological Science Press, Beijing.

Hance, L., Laloux, M., Muchez, P., Groessens, E., Peeters, C. \& Poty, E. 1994. An outline of the Moliniacian (Upper Tournaisian-Lower Viséan) in southern Belgium. Introduction to a field excursion in honour of Prof. Dr. Raphaël Conil 12 October 1991. Mémoires de l'Institut Géologique de l'Université Catholique de Louvain 35, 27-50.

Hecker, M. 2002. Correlation of the Dinantian of the East European Platform and Urals with the type area (Belgium), 52-78. In Hills, L.V., Henderson, C.M. \& Bamber, E.W. (eds) Carboniferous and Permian of the World. Canadian Society of Petroleum Geologists, Memoir 19.

HeCKER, M. 2009. Major guide taxa for correlation of the Moscow and Donets basins Dinantian successions with the type area (Belgium), 198-201. In Puchkov, V.N. (ed.) Carboniferous type sections in Russia and potential global stratotypes, southern Urals session. Proceedings of the International Field Meeting "The historical type sections, proposed and potential GSSP of the Carboniferous in Russia”.

Ivanova, R.M. 1988. Calcareous algae of the Viséan stage of the Urals. In Chuvashov, B.I. \& PuchKov, B.N. (eds) Biostratigraphy and lithology of the Late Palaeozoic of the Urals. Akademiya nauk SSSR, Uralskoe otdelenye, Sbornik nauchnykh trudov. [in Russian]

Ivanova, R.M. \& Bogush, O.I. 1992. Algae as indicators of a biogeographical zonation in the Early Carboniferous of the Urals, Siberia and Northeast Russia. Facies 27, 235-244. DOI 10.1007/BF02536815

JAMES, N.P. 1997. The cool-water carbonate depositional realm, 1-20. In JAmes, N.J. \& Clarke, J. (eds) Cool-water carbonates. SEPM, Special Publication 56. DOI 10.2110/pec.97.56.0001

Kagarmanov, A.K. \& Donakova, L.M. 1990. Decisions of the regional, stratigraphical, interdepartmental conference on the Middle and Late Palaeozoic, Leningrad, 1988, Carboniferous system. 40 pp. VSEGEI, Leningrad. [in Russian]

KalvodA, J. 1983. Preliminary zonation of the Upper Devonian and Lower Carboniferous in Moravia. Miscellanea Micropaleontologica, a memorial volume dedicated to the $18^{\text {th }} \mathrm{Eu}$ ropean Colloquium in Micropaleontology. Knihovnička Zemního plynu a nafty 4, 23-42.

KalvodA, J. 1990. Foraminiferal zonation of the Upper Devonian and Lower Carboniferous in Moravia (Czechoslovakia). Acta Musei Moraviae, Scientiae naturales 75, 71-93.

KalvodA, J. 1999. Tournaisian-Lower Visean calcareous foraminifera: biostratigraphy and palaeogeography. Abhandlungen der geologischen Bundesanstalt 54, 135-145.

Kalvoda, J. 2002. Late Devonian-Early Carboniferous foraminiferal fauna: zonations, evolutionary events, paleobiogeography and tectonic implications. Folia, Geologia 39, 1-213.

Kalvoda, J. 2003. Carboniferous foraminiferal paleobiogeography in Turkey and its implications for plate tectonic reconstructions. Rivista Italiana di Paleontologia i Stratigrafia 109(2), 255-266.
Kalvoda, J., Bábek, O., Devuyst, F.-X. \& Sevastopoulo, G.D. 2011. Biostratigraphy, sequence stratigraphy and gamma-ray spectrometry of the Tournaisian-Viséan boundary interval in the Dublin Basin. Bulletin of Geosciences 86(4), 683-706. DOI 10.3140/bull.geosci.1265

Kalvoda, J., Bábek, O., Fatka, O., Leichmann, J., Melichar, R., NEHYBA, R. \& ŠPAČEK, P. 2008. Brunovistulian terrane (Bohemian Massif, Central Europe) from Late Proterozoic to Late Paleozoic: a review. International Journal of Earth Sciences (Geologische Rundschau) 97, 497-518.

Kalvoda, J., Leichmann, J., BÁbek, O. \& Melichar, R. 2003. Brunovistulian Terrane (Central Europe) and Istanbul Zone (NW Turkey): Late Proterozoic and Paleozoic tectonostratigraphic development and paleogeography. Geologica Carpathica 54, 139-152.

KAYA, O. 1969. Karbon bei Istanbul. Neues Jahrbuch für Geologie und Paläontologie, Monatshefte 1969(3), 160-173.

KAYA, O. 1971. İstanbul'un Karbonifer stratigrafisi. Turkiye Jeoloji Kurumu Bülteni 14(2), 143-199.

KAYA, O. \& MAMET, B. 1971. Biostratigraphy of the Viséan Cebeciköy limestone near İstanbul, Turkey. Journal of Foraminiferal Research 1(2), 77-81. DOI 10.2113/gsjfr.1.2.77

KoRN, D. 1997. Evolution of the Goniatitaceae and ViséanNamurian biogeography. Acta Paleontologica Polonica 42(2), 177-199.

Kulagina, E.I., Gibshman, N.B. \& Pazukhin, V.N. 2003. Foraminiferal zonal standard for the Lower Carboniferous of Russia and its correlation with the conodont zonation. Rivista Italiana di Paleontologia e Stratigrafia 109(2), 173-185.

Kulagina, E.I., KochetKova, N.M. \& Byvsheva, T.B. 1988. On the palaeontological characteristics of the early-middle Viséan deposits of Orenburg. In Tchibrikova, Y.V. (ed.) Biostratigraphy of the Devonian and Carboniferous of the Urals. Bashkirian Scientific Centre, Urals Division of the Academy of Sciences of the USSR. [in Russian]

Leven, E.Y. \& OKaY, A.I. 1996. Foraminifera from the exotic Permo-Carboniferous limestone blocks in the Karakaya complex, northwestern Turkey. Rivista Italiana di Paleontologia e Stratigrafia 102(2), 139-174.

LIPINA, O.A. 1955. Foraminifers of the Tournaisian stage and of the upper part of the Devonian from the Volga-Ural area and from the western slope of central Urals. Trudy Instituta Geologii, Geologichevskaya seriya 163(70), 1-96. [in Russian; French translation: BRGM 1640]

Lys, M. 1988. Biostratigraphie du Carbonifère en Mésogée (Espagne, Afrique du Nord, Régions Egéennes, Proche-Orient). Etudes micropaléontologiques (Foraminifères) paléobiogéographie. Documents du B.R.G.M. 147, 1-315.

MalaKhova, N.P. 1975a. Foraminifers from the early Viséan of the western slope of southern Urals, 5-70. In Malakhova, N.P. \& Chuvashov, B.I. (eds) Foraminifery $i$ stratigrafiya rannego vize Urala (Foraminifers and stratigraphy from the early Viséan of Urals). Trudy Instituta Geologii i Geokhimii 112. [in Russian]

MalaKhOVA, N.P. 1975b. Foraminifers, algae and stratigraphy of the early Viséan of the western slope of southern Urals, 71-109. In Malakhova, N.P. \& Chuvashov, B.I. (eds) Foraminifery i stratigrafiya rannego vize Urala (Foraminifers and stratigraphy from early Viséan of Urals). Trudy Instituta Geologii i Geokhimii 112. [in Russian] 
MAMET, B. 1973. Foraminiferal biostratigraphy of the Lower Carboniferous Trakya and Heybeliada formations, Istanbul region, Turkey. In Kaya, O. (ed.) Paleozoic of Istanbul. Ege Universitesi, Fen Fakültesi, Kitaplar Serisi 40.

MAMET, B. 1974. Une zonation par foraminifères du Carbonifère inférieur de la Téthys Occidentale. Comptes Rendus du $7 e$ Congrès International de Stratigraphie et Géologie du Carbonifère 3, 391-408.

Mamet, B. \& Roux, A. 1981. Note sur le genre Issinella (algue verte paléozoïque). Revue de Micropaléontologie 23(3-4), 151-158.

Marfenkova, M.M. 1991. Marine Carboniferous from Kazakhstan; stratigraphy, zonal sequence, foraminifers. $199 \mathrm{pp}$. Gylym, Alma-Ata. [in Russian]

Meinhold, G., Reischmann, T., Kostopoulos, D., Lehnert, O., Makutov, D. \& Sergeev, S. 2008. Provenance of sediments during subduction of Palaeotethys: Detrital zircon ages and olistolith analysis in Palaeozoic sediments from Chios Island, Greece. Palaeogeography, Palaeoclimatology, Palaeoecology 263, 71-91. DOI 10.1016/j.palaeo.2008.02.013

Menning, M., Alekseev, A.S., Chuvashov, B.I., Davydov, V.I., Devuyst, F.-X., Forke, H.C., Grunt, T.A., Hance, L., Heckel, P.H., Izokh, N.G., Jin, Y.G., Jones, P.J., Kotlyar, G.V., Kozur, H.W., Nemyrovska, T.I., Schneider, J.W., Wang, X.D., Weddige, K., Weyer, D. \& Work, D.M. 2006. Global time scale and regional stratigraphic reference scales of Central and West Europe, East Europe, Tethys, South China, and North America as used in the Devonian-Carboniferous-Permian correlation chart (DCP 2003). Palaeogeography, Palaeoclimatology, Palaeoecology 240, 318-372.

Mikhno, N.M. \& BALAKIN, G.V. 1975. Foraminifera and bryozoa from the Early Carboniferous Chatkal horizon. Izdatelstvo "Fan", Uzbekskoy SSR, Tashkent. [in Russian]

Moix, P., Beccaletto, L., Kozur, H.W., Hochard, C., Rosselet, F. \& StAmpfli, G.M. 2008. A new classification of the Turkish terranes and sutures and its implication for the paleotectonic history of the region. Tectonophysics 451(1-4), 7-39. DOI 10.1016/j.tecto.2007.11.044

Moix, P., Beccaletto, L., Masset, O., Kozur, H.W., Dumitrica, P., Vachard, D., Martini, R. \& Stampfli, G.M. 2011. Geology and correlation of Mersin Mélanges, southern Turkey. Turkish Journal of Earth Sciences 20, 57-98.

Noble, P.J., Tekin, U.K., Gedik, I. \& Pehlivan, Ș. 2008. Middle to upper Tournasian (sic) radiolaria of the Baltalimanı Formation, Istanbul, Turkey. Journal of Paleontology 82(1), 37-56.

OKAY, A.I. \& TÜYsüz, O. 1999. Tethyan sutures of northern Turkey, 475-515. In Durand, B., Jolivet, L., Horváth, F. \& SÉRAnNe, M. (eds) The Mediterranean Basins: Tertiary extension within the Alpine Orogen. Geological Society of London, Special Publication 156.

OKay, A.I., Noble, P.J. \& Tekin, U.K. 2011. Devonian radiolarian ribbon cherts from the Karakaya Complex, Northwest Turkey: Implications for the Paleo-Tethyan evolution. Comptes Rendus Palevol 10,1-10.

OKuyucu, C. \& VAchaRd, D. 2006. Late Viséan foraminifera and algae from the Cataloturan Nappe, Aladag Mountains, eastern Taurides, southern Turkey. Geobios 39, 535-554.

DOI 10.1016/j.geobios.2005.03.006

ÖNALAN, M. 1981. İstanbul Ordovisiyen ve Silüriyen istifinin çökelme ortamları. İstanbul Üniversitesi Mühendislik Fakültesi Dergisi 2, 161-177.

ÖzGÜL, N. 2012. Stratigraphy and some structural features of the İstanbul Palaeozoic. Turkish Journal of Earth Sciences 21, 817-866.

ÖZgÜL, N., ÜNer, K., AKMEȘE, İ., BILGIN, İ., KoKUZ, R., ÖZCAN, İ., Yildiz, Z., Yildirim, Ü., AkdaĞ, Ö. \& Tekin, M. 2005. Ístanbul il alanının genel jeoloji özellikleri. İstanbul Büyükșehir Belediyesi Planlama ve İmar Daire Baskanlığı.

Penck, W. 1919. Grundzüge der Geologie des Bosphorus. Veröffentlichungen des Instituts für Meereskunde an der Universität Berlin, n. s. 4, 1-71.

Pille, L. 2008. Foraminifères et algues calcaires du Mississippien supérieur (Viséen supérieur-Serpukhovien): rôles biostratigraphique, paléoécologique et paléogéographique aux échelles locale, régionale et mondiale. $\mathrm{PhD}$ thesis, University of Lille 1.

Pille, L., Vachard, D., Argyriadis, I. \& Aretz, M. 2010. Revision of the late Visean-Serpukhovian (Mississippian) calcareous algae, foraminifers and microproblematica from Balia Maden (NW Turkey). Geobios 43(5), 531-546.

DOI 10.1016/j.geobios.2010.03.005

Poty, E., Devuyst, F.-X. \& Hance, L. 2006. Upper Devonian and Mississippian foraminiferal and rugose coral zonations of Belgium and Northern France, a tool for Eurasian correlations. Geological Magazine 143(6), 829-857.

DOI 10.1017/S0016756806002457

Rauzer-Chernousova, D.M. 1948. Materialy k faune foraminifer kamennougolnykh otlozhenii Tsentralnogo Kazakhstana [Materials for foraminiferal fauna from the Carboniferous deposits from central Kazakhstan]. Trudy Instituta geologicheskikh nauk, Geologicheskaya seriya 66(21), 1-66. [in Russian]

Reituinger, E.A. 1954. Devonian foraminifers from the eastern part of the Russian Platform. Paleontologicheskii sbornik VNIGRI 1, 41-51. [in Russian]

ReitLinger, E.A. 1950. Foraminifera from Middle Carboniferous deposits of the central part of the Russian Platform (excepting the family Fusulinidae). Trudy Instituta geologicheskikh nauk, Geologicheskaya seriya 126(47), 1-126. [in Russian, French translation: BRGM 1456]

SAmANKASsou, E. 2002. Cool-water carbonates in a paleoequatorial shallow-water environment: The paradox of the Auernig cyclic sediments (Upper Pennsylvanian, Carnic Alps, Austria-Italy) and its implications. Geology 30(7), 655-658. DOI 10.1130/0091-7613(2002)030<0655:CWCIAP > 2.0.CO;2

Strickland, H.E. 1840. On the geology of the Thracian Bosphorus. Transactions of the Geological Society of London 2, 5(2), 385-391.

TChinatchefF, P. 1854. Dépôts paléozoïques de la Cappadoce et du Bosphore. Bulletin de la Société géologique de France 11, 402-416.

Termier, G., Termier, H. \& Vachard, D. 1977. Sur l'âge des faunes marines froides corrélatives des glaciations gondwanes du Carbonifère. Comptes Rendus de l'Académie des Sciences de Paris 284, 2321-2322.

VACHARD, D. 1977. Etude stratigraphique et micropaléontologique (algues et foraminifères) du Viséen de la Montagne Noire (Hérault, France). Mémoires de l'Institut Géologique de l'Université de Louvain 29, 111-195. 
VACHARD, D. 1994. Révision du genre Luteotubulus Vachard, 1977 (pseudo-algae issinellide), grâce au matériel de R. Conil. Mémoires de l'Institut Géologique de l'Université Catholique de Louvain 35, 213-219.

VACHARD, D. \& CLÉMENT, B. 1994. L'Hastarien (ex-Tournaisien inférieur et moyen) à algues et foraminifères de la Zone Pélagonienne (Attique, Grèce). Revue de Micropaléontologie 37(4), 289-319.

VACHARD, D. \& Cózar, P. 2010. An attempt of classification of the Palaeozoic incertae sedis Algospongia. Revista Española de Micropaleontología 42(2), 129-241.

Vachard, D., Pille, L., Aretz, M., Herbig, H.G. \& Cózar, P. 2006. Eovelebitella (dasyclad algae) and importance of its Brigantian subprovince within the western Palaeotethys. Kölner Forum für Geologie und Paläontologie 15, 120-121.

VAchard, D., Termier, H. \& Termier, G. 1977. Sur la transgression viséenne au Jebel Goulib (Maroc Central). Bulletin mensuel de la Société linnéenne de Lyon 46(8), 267-281.

Vdovenko, M.V. 1980. Viséan stage; zonal subdivisions and palaeozoogeographical provinces. Naukova dumka, Kiev. [in Russian]

Vdovenko, M.V. 1986. Rannekammenougolnye foraminifery Pridobrudzhinskogo Progiba [Early Carboniferous foraminifers from the Predobrudzha area]. Bulgarska Academiya na Naukite, Paleontologiya, Stratigrafiya i Litologiya 22, 3-14. [(in Russian]

VDOVEnKo, M.V. 2001. Atlas of Foraminifera from the Upper Viséan and Lower Serpukhovian (Lower Carboniferous) of the Donets Basin (Ukraine). Abhandlungen und Berichte für Naturkunde 23, 93-178.

VERNEUIL, M. DE 1836-1837. Notice géologique sur les environs de Constantinople. Bulletin de la Société géologique de France 1(8), 268-278.

Weyer, D. 2006. 5. Faunen des deutschen Unterkarbons; 5.1 Korallen, 69-88. In Deutsche Stratigraphische Kommission (ed.) Stratigraphie von Deutschland, VI. Unterkarbon (Mississippium). Schriftenreihe der Deutschen Gesellschaft für Geowissenschaften 41.

YALÇINLAR, I. 1951. İstanbul civarının Paleozoik arazisine yeni musahedeler. Türkiye Jeoloji Kurumu Bülteni 3(1), 125-130.

YALÇINLAR, I. 1954. Sur la présence de schistes carbonifères et de plantes fossiles à l'W d'Istanbul. Comptes Rendus Sommaires de la Société géologique de France 2, 23-25.

YANEv, S. 1993. Gondwana Paleozoic terranes in the Alpine collage system of the Balkans. Himalayan Geology 4(2), 257-270.

YAnev, S. 1997. Paleozoic migration of terranes from the basement of the eastern part of the Balkan peninsula from periGondwana to Laurussia. Turkish Association Petroleum Geologists, Special Publication 3, 89-100.

Yanev, S. 2000. Palaeozoic terranes of the Balkan Peninsula in the framework of Pangea assembly. Palaeogeography, Palaeoclimatology, Palaeoecology 161, 151-177.

DOI 10.1016/S0031-0182(00)00121-8

YANEV, S. \& AdAmia, S. 2010. General correlation of the Late Palaeozoic sequences in the Balkans and the Caucasus. Yerbilimleri 31(1), 1-22.

Yanev, S., GöncÜoĞLU, M.C., Gedik, I., Lakova, I., BonCheva, I., Sachanski, V., Okuyucu, C., Özgül, N., Timur, E. \& Maliakov, Y. 2006. Stratigraphy, correlations and palaeogeography of Palaeozoic terranes in Bulgaria and NW Turkey: a review of recent data. In Robertson, A.H.F., Mountrakis, D. $\&$ BRUn, J.-P. (eds) Tectonic Evolution of the Eastern Mediterranean. Geological Society of London, Special Publications 260.

Yilmaz, Y. 1991. Allochthonous terranes in the Tethyan Middle East: Anatolia and the surrounding regions. In DEwEY, J.F., Gass, I.G., Curry, G.B., Harris, N.B.W. \& ȘENGÖr, C. (eds) Allochthonous terranes. Cambridge University Press, Cambridge. 